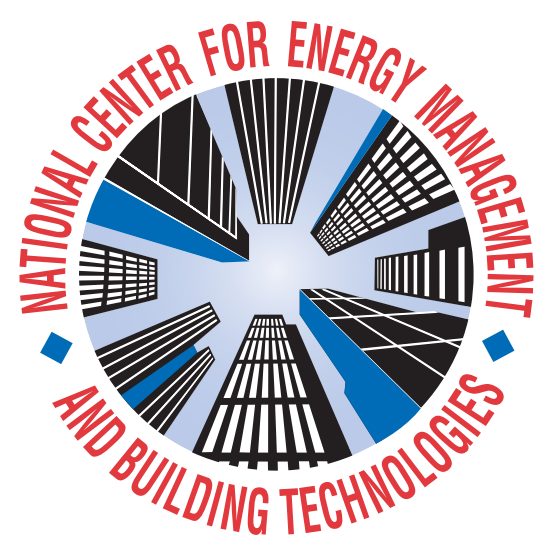

FINAL REPORT NCEMBT-090921

\title{
STATISTICAL ANALYSIS AND INTERPRETATION OF BUILDING CHARACTERIZATION, INDOOR ENVIRONMENTAL QUALITY MONITORING AND ENERGY USAGE DATA FROM OFFICE BUILDINGS AND CLASSROOMS IN THE UNITED STATES
}

\section{SEPTEMBER 2009}

Linda D. Stetzenbach, Ph.D. and Lauren Nemnich, M.A. University of Nevada Las Vegas

Davor Novosel

National Center for Energy Management and Building Technologies

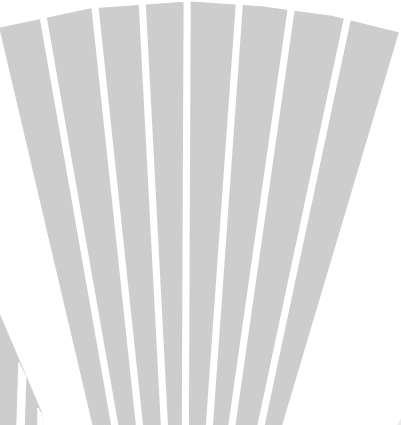


FINAL REPORT NCEMBT-090921

\section{NATIONAL CENTER FOR ENERGY MANAGEMENT AND BUILDING TECHNOLOGIES TASK 08-01: STATISTICAL ANALYSIS AND INTERPRETATION OF BUILDING CHARACTERIZATION, INDOOR ENVIRONMENTAL QUALITY MONITORING AND ENERGY USAGE DATA FROM OFFICE BUILDINGS AND CLASSROOMS IN THE UNITED STATES}

SEPTEMBER 2009

Prepared By:

Linda D. Stetzenbach, Ph.D. and Lauren Nemnich, M.A.

University of Nevada Las Vegas

Davor Novosel

National Center for Energy Management and Building Technologies

Prepared For:

U.S. Department of Energy

Rob Martinez

Project Officer

This report was prepared for the U.S. Department of Energy

Under Grant DE-FG26- 08NT02119 


\section{NOTICE}

This report was prepared as an account of work sponsored by an agency of the United States government. Neither the United States government nor any agency thereof, nor any of their employees, makes any warranty, express or implied, or assumes any legal liability or responsibility for the accuracy, completeness, or usefulness of any information, apparatus, product, or process disclosed, or represents that its use would not infringe privately owned rights. Reference herein to any specific commercial product, process, or service by trade name, trademark, manufacturer, or otherwise does not necessarily constitute or imply its endorsement, recommendation, or favoring by the United States government or any agency thereof. The views and opinions of authors expressed herein do not necessarily state or reflect those of the United States government or any agency thereof.

\section{UNIVERSITY OF NEVADA, LAS VEGAS, CONTACT}

Linda D. Stetzenbach, Ph.D.

Professor, Department of Environmental and Occupational Health

School of Public Health

University of Nevada, Las Vegas

4505 South Maryland Parkway

Las Vegas, NV 89154-4009

(702) 895-5509

linda.stetzenbach@unlv.edu

\section{National Center for Energy Management and BuILding TeChnologies Contact}

Davor Novosel

Chief Technology Officer

National Center for Energy Management and Building Technologies

601 North Fairfax Street, Suite 250

Alexandria VA 22314

703-299-5633

dnovosel@ncembt.org

www.ncembt.org 


\section{TABLE OF CONTENTS}

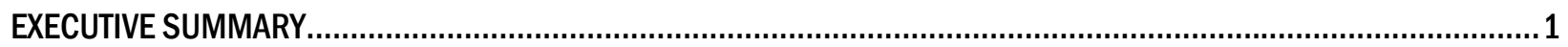

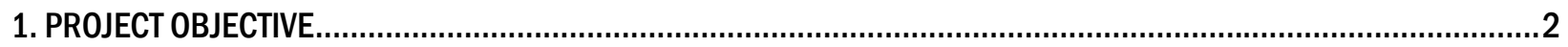

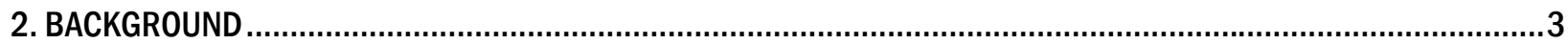

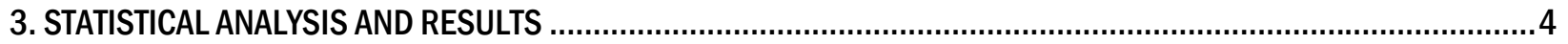

3.1 Enhanced Collection of Energy and Building Characterization Data ............................................................. 4

3.2 IEQ

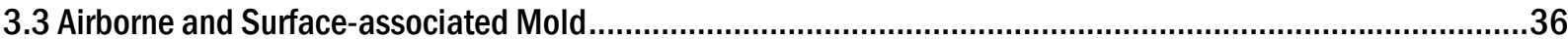

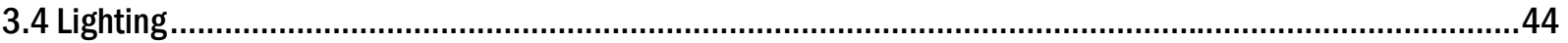

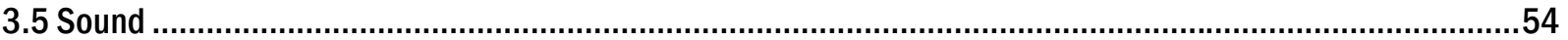

4. INTERPRETATION OF THE DATA

4.1 IEQ

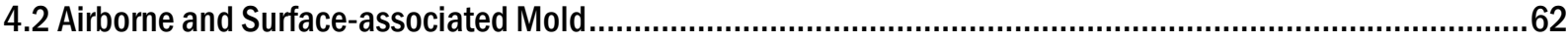

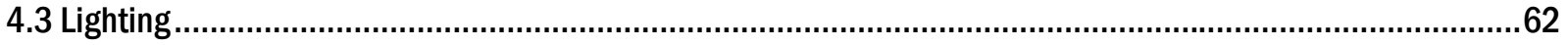

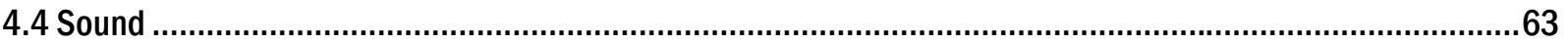

5. JOURNAL ARTICLE

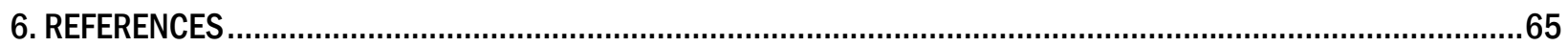




\section{LIST OF TABLES}

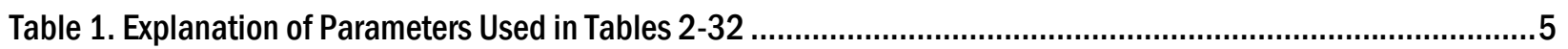

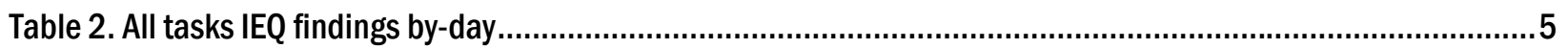

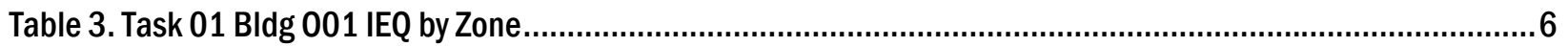

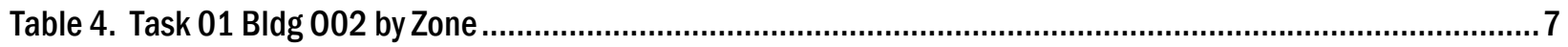

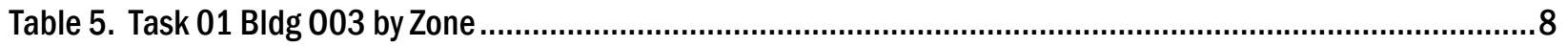

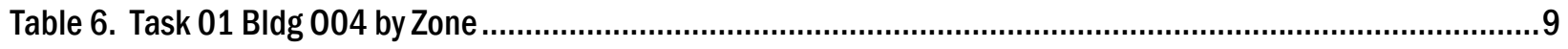

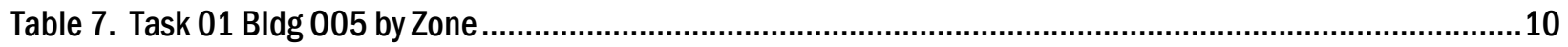

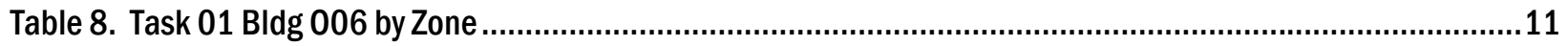

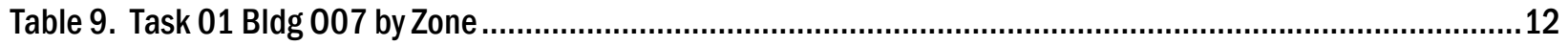

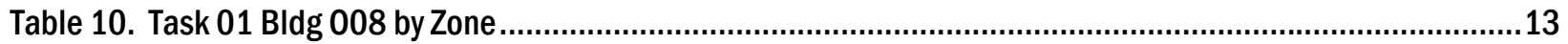

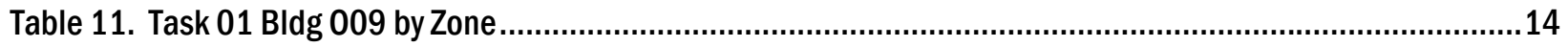

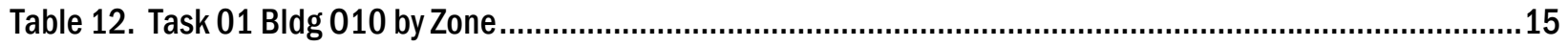

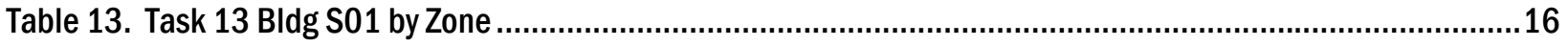

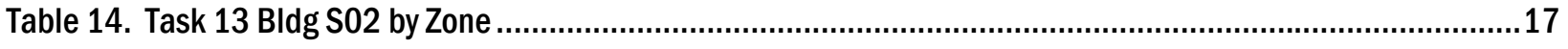

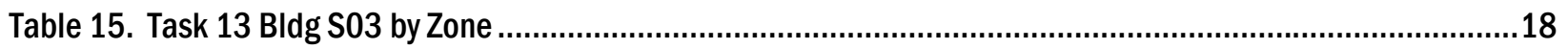

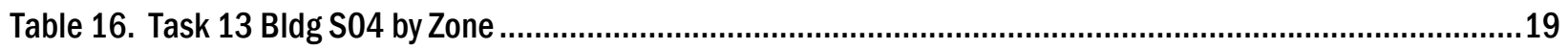

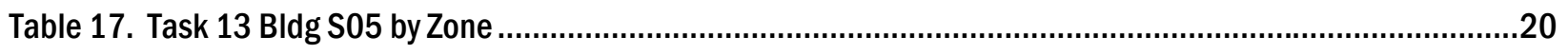

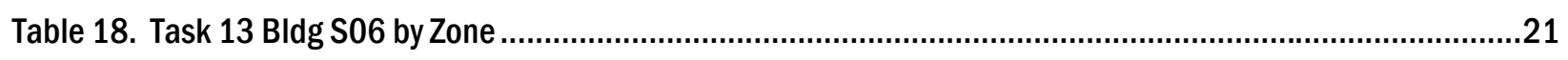

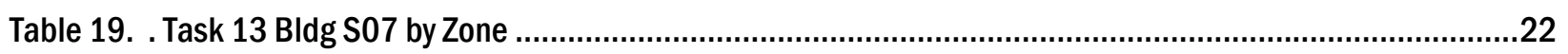

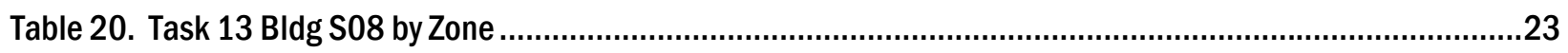

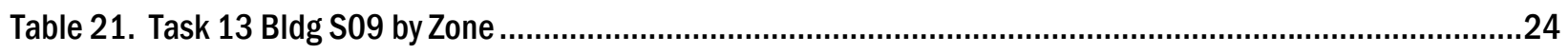

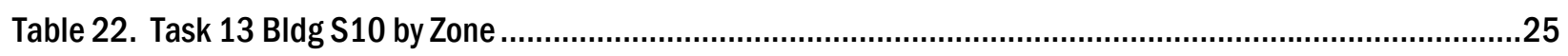

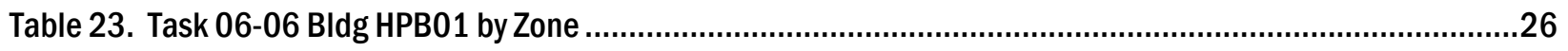

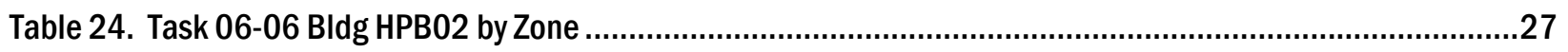

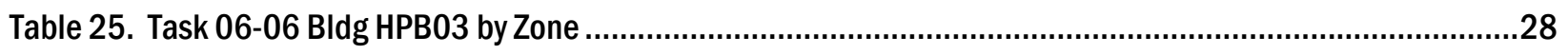

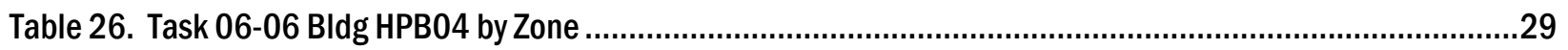

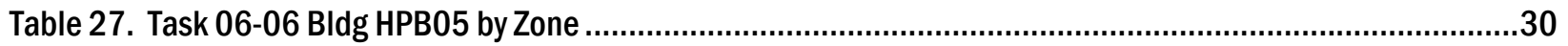

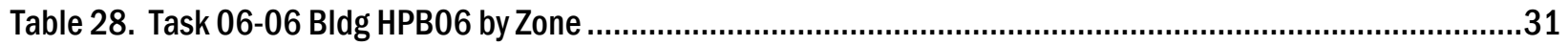

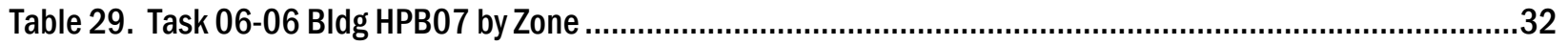

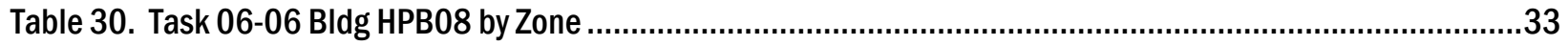

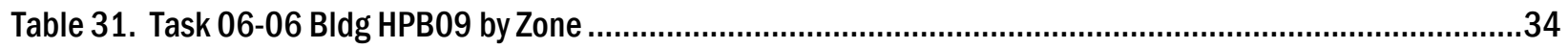

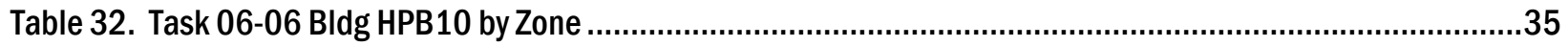

Table 33. . Statistical Results by Day for All Tasks for Airborne Culturable Fungi, Airborne Non-culturable Spores, and

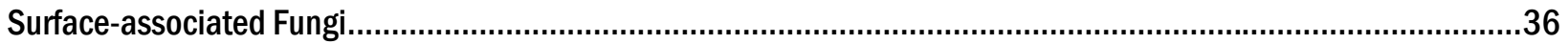


Table 34. Task 1 Office Buildings by Zone Statistical Results for Airborne Non-culturable Spores

Table 35. Task 10 Office Buildings by Zone Statistical Results for Surface-associated Fungi...................................38

Table 36. Task 13 Schools by Zone Results for Airborne Culturable Fungi..........................................................39

Table 37. Task 13 Schools by Zone Results for Airborne Non-culturable Spores .................................................40

Table 38. Task 13 Schools by Zone Findings for Surface-associated Fungi ......................................................41

Table 39. Task 0606 High Performance Buildings by Zone Results for Airborne Culturable Fungi ...........................42

Table 40. Task 0606 High Performance Buildings by Zone Results for Airborne Non-culturable Spores .................43

Table 41. Task 0606 High Performance Buildings by Zone Results for Surface-associated Fungi ..........................44

Table 42. Task 10 Office Buildings Lighting by Zone: All Buildings......................................................................45

Table 43. Task 10 Office Buildings Lighting by Zone: Building 001 ..............................................................46

Table 44. Task 10 Office Buildings Lighting by Zone: Building 002 ................................................................47

Table 45. Task 10 Office Buildings Lighting by Zone: Building 004 .................................................................48

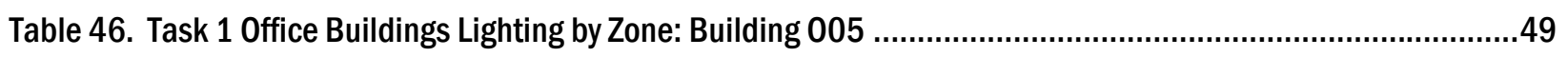

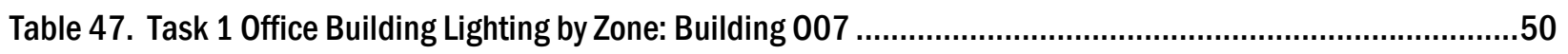

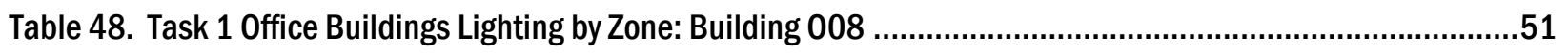

Table 49. Task 1 Office Building Lighting by Zone: Building 009 ...................................................................52

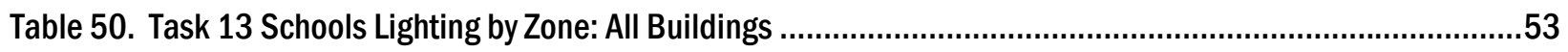

Table 51. Task 0606 High Performance Lighting by Zone: All Buildings ........................................................54

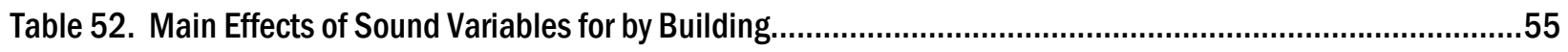

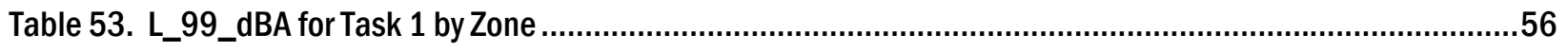

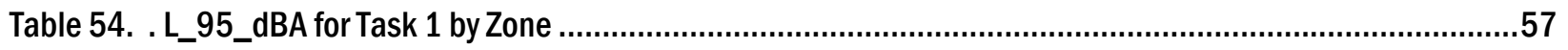

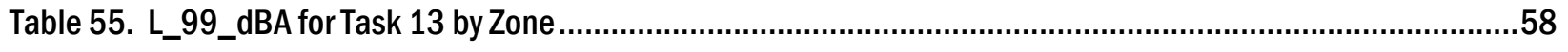

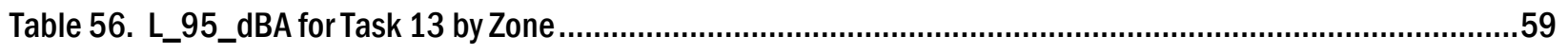

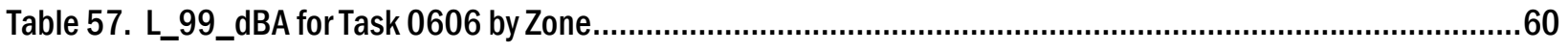

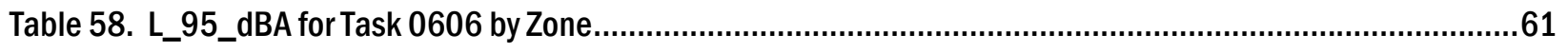


NCEMBT-090921 


\section{EXECUTIVE SUMMARY}

Three independent tasks had been performed (Stetzenbach 2008, Stetzenbach 2008b, Stetzenbach 2009) to measure a variety of parameters in normative buildings across the United States. For each of these tasks 10 buildings were selected as normative indoor environments. Task 1 focused on office buildings, Task 13 focused on public schools, and Task 0606 focused on high performance buildings. To perform this task it was necessary to restructure the database for the Indoor Environmental Quality (IEQ) data and the Sound measurement as several issues were identified and resolved prior to and during the transfer of these data sets into SPSS. During overview discussions with the statistician utilized in this task it was determined that because the selection of indoor zones (1-6) was independently selected within each task; zones were not related by location across tasks. Therefore, no comparison would be valid across zones for the 30 buildings so the by location (zone) data were limited to three analysis sets of the buildings within each task. In addition, differences in collection procedures for lighting were used in Task 0606 as compared to Tasks $01 \& 13$ to improve sample collection. Therefore, these data sets could not be merged and compared so effects by-day data were run separately for Task 0606 and only Task $01 \& 13$ data were merged.

Results of the statistical analysis of the IEQ parameters show statistically significant differences were found among days and zones for all tasks, although no differences were found by-day for Draft Rate data from Task 0606 ( $>0.05$ ). Thursday measurements of IEQ parameters were significantly different from Tuesday, and most Wednesday measures for all variables of Tasks $1 \& 13$. Data for all three days appeared to vary for Operative Temperature, whereas only Tuesday and Thursday differed for Draft Rate 1m. Although no Draft Rate measures within Task 0606 were found to significantly differ by-day, Temperature measurements for Tuesday and Thursday showed variation. Moreover, Wednesday measurements of Relative Humidity within Task 0606 varied significantly from either Tuesday or Thursday. The majority of differences in IEQ measurements by-zone were highly significant $(\mathrm{p}<0.001)$, with the exception of Relative Humidity in some buildings.

When all task data were combined (30 buildings) neither the airborne culturable fungi nor the airborne non-culturable spore data differed in the concentrations found at any indoor location in terms of day of collection. However, the concentrations of surface-associated fungi varied among the day of collection. Specifically, there was a lower concentration of mold on Tuesday than on Wednesday, for all tasks combined. As expected, variation was found in the concentrations of both airborne culturable fungi and airborne non-culturable fungal spores between indoor zones (1-6) and the outdoor zone (zone 0). No variation was found among the indoor zones of office buildings for Task 1 in the concentrations of airborne culturable fungi. However, airborne non-culturable spores did vary among zones in one building in Task 1 and variation was noted between zones in surface-associated fungi.

Due to the lack of multiple lighting measurements for Tasks 13 and 0606, by-day comparisons were only performed for Task 1. No statistical differences were observed in lighting with respect to the day of collection. There was a wide range of variability by-zone among seven of the office buildings. Although few differences were found for the brightest illumination of the worksurface (IllumWkSfcBrtst) and the darkest illumination of the worksurface (IllumWkSfcDrkst) in Task 1, there was considerable variation for these variables in Task 13 and Task $0606(\mathrm{p}<0.001)$. Other variables that differed by-zone in Task 13 include CombCCT and AmbCCT1 for S03, S07, and S08. Additionally, AmbChromX1, CombChromY, and CombChromX varied by-zone for school buildings S02, S04, and S05, respectively.

Although all tasks demonstrated significant differences in sound measurements by zone, some of the buildings within each task did not appear to differ in sound quality. Hence, post-hoc tests were not appropriate and individual zones were not compared for these buildings. It is interesting to note that sound measurements in some buildings were widely varied with most zone comparisons and other buildings varied between only a few zones. 


\section{PROJECT OBJECTIVE}

This research effort focused on statistical analysis and interpretation of the previously collected data under NCEMBT Task 1, 13 and 06-06. This task also included compiling information that was not collected during the monitoring of the previous buildings (e.g., additional building characterization and energy usage), and refining the NCEMBT database capabilities to result in a readily searchable/data mining resource for researchers interested in minimizing energy usage while providing quality indoor environments for occupants. The goals of this task included providing statistical interpretation of the existing NCEMBT-sponsored monitoring data to provide new understanding of the relationship of indoor environmental quality parameters to energy usage and building characteristics, and provide additional information from the building managers/facilities personnel regarding energy usage and building characteristics not obtained during the monitoring projects. Comparison and correlation of the data, and interpretation of the results were vetted through an expert advisory board. The results of this project will be made available for downloading from the Building Sciences Database on NCEMBT's website at www.ncembt.org. 


\section{BACKGROUND}

Three previous studies (Task 1, Measurement and Verification of Building Performance Characteristics; Task 13, Building Performance Characterization, Energy Usage, and Indoor Environmental Quality in Educational Facilities; and Task 06-06, Energy Performance and Environmental Characteristics of High Performance Buildings) were sponsored by the National Center for Energy Management and Building Technologies (NCEMBT) under DOE award DE-FC26-03GO13072. These three tasks provided data in typical office buildings, schools, and high performance buildings and some energy consumption data. The data included thermal comfort (i.e., temperature, relative humidity, and draft), carbon dioxide $\left(\mathrm{CO}_{2}\right)$, volatile organic compounds (VOCs), sound, lighting, and airborne and surface-associated mold. All buildings were selected based on building design and construction characteristics as determined by a series of screening questions answered by the buildings districts' facilities department personnel. This included the physical characteristics of the building, the age of structure, its geographical location, and type of mechanical air handling system. In each building six indoor areas (zones/locations) were selected that were representative of the building's configuration. Measurement instrumentation was located in each of the six indoor zones. Additionally, an outdoor location at each building was selected as a control site for some of the measurements. Separate reports have been submitted presenting the data from the three independent surveys, but the number of buildings in each survey was limited to 10 by the US DOE. The study objective for this task was to combine data from the 30 buildings which would permit raising the number of measurements and increase the power of the statistical analysis. Therefore, this task was conducted to investigate whether there were differences among days of data collection (Tuesday, Wednesday, or Thursday) and/or differences in the variables of interest among the zones for each building (zones $0-6$, with $0=$ outdoor measure) within each task. 


\section{STATISTICAL ANALYSIS AND RESULTS}

This task was focused on sophisticated statistical analysis of the measurement data (i.e., thermal comfort [temperature, relative humidity, and draft], carbon dioxide $\left[\mathrm{CO}_{2}\right]$, volatile organic compounds [VOCs], sound, lighting, and airborne and surface-associated mold) from the 30 buildings. Previous analysis was limited to three separate clusters of 10 buildings each. It was envisioned that the results from the 30 buildings would strengthen statistical power of the dataset. Specific statistical methods used for the analysis were determined during the task but were focused on i) descriptive statistics used to determine demographic characteristics both within and between the thirty buildings, ii) homogeneity between building characteristics tested using the chi-squared test for independence, iii) differences in continuous outcome measures such as IEQ measurements and energy usage between buildings using analysis of variance (ANOVA) with post hoc tests where applicable, and iv) relationships between continuous measurements using Spearman's and/or Pearson's correlation coefficients proving a measure of agreement. In addition, transformation or nonparametric techniques were applied as needed following testing for normality of the data. No VOC measurements were positive. Therefore, these data were not subjected to statistical analysis

\subsection{Enhanced Collection OF Energy And BuILDing Characterization Data}

During the previous three tasks building characteristic data were collected from the building facilities managers. However, gaps in building information were found by task advisors Dr. James Woods and Mr. Richard Switzer. Therefore, this task included a component to increase the information from some of the 30 monitored buildings concerning building characteristics not included in the original data collection. Additional data submitted by the facilities managers were entered into the database as they were received.

\subsection{IEQ}

Prior to conducting statistical comparisons, it was noted that differences in collection procedures for some IEQ measurements were used for Task 0606 as compared to Tasks $1 \& 13$. Therefore, these data sets could not be merged and compared. Therefore, effects by-day data were run separately for Task 0606 but Tasks $1 \& 13$ data were merged. Additionally, it was determined that IEQ Draft Rates in all tasks were non-normally distributed and required non-parametric testing. Hence, all Draft Rate data were analyzed via rank order for both tests by-day and by-zone. Statistical tests were conducted for each of the following different measures: operative temperature (OpTemp) for Tasks $1 \& 13$ and room temperature (Temp) for Task 0606; Relative humidity (RelHumidity); and Draft Rates at $0.1 \mathrm{~m}, 0.6 \mathrm{~m}$, and $1.1 \mathrm{~m}$ (DraftRate0_1m, DraftRate0_6m, DraftRate1_1m, respectively).

Univariate analysis of variance (ANOVA) was used to determine the presence or absence of a main effect among the three days or the seven zones. If a main effect was observed, post-hoc analysis was performed to determine which specific days or zones differed through either Bonferroni's test or Dunnett's T3 test for assumption of equal variance or unequal variance, respectively. As shown in the following tables (Table 1-32), significant differences were found among days and zones for all tasks, although no differences $(\mathrm{p} \geq 0.05)$ were found by-day for Draft Rate data from Task 0606 . 
Table 1. Explanation of Parameters Used in Tables 2-32

\begin{tabular}{l} 
Parameter Name in \\
Tables 2 through 32 \\
\hline Temp \\
\hline OpTemp \\
\hline RelHumidity \\
\hline DraftRatex_ym
\end{tabular}

\begin{tabular}{|l|c|}
\hline Parameter Meaning & Unit \\
\hline Dry bulb air temperature at $0.6 \mathrm{~m}$ above the floor & ${ }^{\circ} \mathrm{C}$ \\
\hline Operative Temperature measured at $0.6 \mathrm{~m}$ above the floor & ${ }^{\circ} \mathrm{C}$ \\
\hline Relative Humidity measured at $0.6 \mathrm{~m}$ above the floor & $\%$ \\
\hline $\begin{array}{l}\text { Draft rate measured at three heights, i.e. } 0.1 \mathrm{~m}, \mathbf{0 . 6} \mathrm{m} \text { and } 1.1 \mathrm{~m} \\
\text { above the floor. }\end{array}$ & $\mathrm{fpm}$ \\
\hline
\end{tabular}

Table 2. All tasks IEQ findings by-day

\begin{tabular}{|c|c|c|c|c|c|}
\hline \multicolumn{6}{|c|}{ Task $01 \& 13$} \\
\hline \multirow[t]{2}{*}{ Comparison } & \multicolumn{5}{|c|}{ Variable } \\
\hline & OpTemp & RelHumidity & DraftRate0_1m & DraftRate0_6m & DraftRate1_1m \\
\hline & $(d f=2,6168)$ & $(d f=2,6291)$ & $(d f=2,6135)$ & $(d f=2,6189)$ & $(d f=2,5869)$ \\
\hline Main Effect & $\begin{array}{c}F=22.409 \\
p<0.001\end{array}$ & $\begin{array}{c}F=14.583 \\
p<0.001\end{array}$ & $F=7.471 ; p<0.05$ & $\begin{array}{c}F=7.854 \\
p<0.001\end{array}$ & $\begin{array}{c}F=10.312 \\
p<0.001\end{array}$ \\
\hline Tue vs. Wed & $p<0.05$ & n.s. & n.s. & n.s. & n.s. \\
\hline Wed vs. Thu & $p<0.001$ & $p<0.001$ & n.s. & $p<0.05$ & $p<0.001$ \\
\hline Tue vs. Thu & $p<0.001$ & $p<0.001$ & $p<0.001$ & $p<0.001$ & $p<0.001$ \\
\hline \multicolumn{6}{|c|}{ *RelHumidity \& Draft Rate 1_1m Post-Hoc via Dunnett T3; OpTemp \& DraftRate0_1m via Bonferroni } \\
\hline \multicolumn{6}{|c|}{ *Draft Rates ranked prior to analysis for non-parametric measures } \\
\hline \multicolumn{6}{|c|}{ Task 06-06 } \\
\hline \multirow[t]{3}{*}{ Comparison } & \multicolumn{5}{|c|}{ Variable } \\
\hline & Temp & RelHumidity & DraftRate0_1m & DraftRate0_6m & DraftRate1_1m \\
\hline & $(d f=2,2796)$ & $(d f=2,2796)$ & $(d f=2,2406)$ & $(d f=2,2297)$ & $(d f=2,2370)$ \\
\hline Main Effect & $F=6.443 ; p<0.01$ & $\begin{array}{c}F=9.250 \\
p<0.001\end{array}$ & n.s. & n.s. & n.s. \\
\hline Tue vs. Wed & n.s. & $p<0.01$ & & & \\
\hline Wed vs. Thu & n.s. & $p<0.001$ & & & \\
\hline Tue vs. Thu & $p<0.01$ & n.s. & & & \\
\hline \multicolumn{6}{|c|}{ *RelHumidity Post-Hoc via Dunnett T3; Temp via Bonferroni } \\
\hline *Draft Rates & prior to analysis & -parametric r & & & \\
\hline
\end{tabular}


Table 3. Task 01 Bldg 001 IEQ by Zone

\begin{tabular}{|c|c|c|c|c|c|}
\hline \multirow[t]{2}{*}{ Zone Comparison } & \multicolumn{5}{|c|}{ Variable } \\
\hline & OpTemp & RelHumidity & DraftRate0_1m & DraftRate0_6m & DraftRate1_1m \\
\hline Main Effect & $\begin{array}{c}F=43.504 \\
p<0.001\end{array}$ & $\begin{array}{c}F=2.694 \\
p=0.021\end{array}$ & $\begin{array}{c}F=213.518 \\
p<0.001\end{array}$ & $\begin{array}{c}F=100.472 \\
p<0.001\end{array}$ & $\begin{array}{c}F=54.583 \\
p<0.001\end{array}$ \\
\hline $1 \mathrm{v} 2$ & $p<0.001$ & & $p<0.001$ & & $p<0.001$ \\
\hline $1 v 3$ & $p<0.001$ & & $p<0.001$ & & $p<0.001$ \\
\hline $1 v 4$ & $p<0.001$ & & $p<0.001$ & $p<0.001$ & $p=0.013$ \\
\hline $1 v 5$ & $p<0.001$ & & $p<0.001$ & $p<0.001$ & $p<0.001$ \\
\hline $1 v 6$ & $p=0.028$ & & $p<0.001$ & $p<0.001$ & $p<0.001$ \\
\hline $2 v 3$ & $p<0.001$ & & $p=0.014$ & & \\
\hline $2 v 4$ & $p<0.001$ & & & $p<0.001$ & $p<0.001$ \\
\hline $2 v 5$ & & & $p<0.001$ & $p<0.001$ & \\
\hline $2 v 6$ & & & $p<0.001$ & $p<0.001$ & \\
\hline $3 v 4$ & & & & $p<0.001$ & $p<0.001$ \\
\hline $3 v 5$ & $p<0.001$ & & $p<0.001$ & $p<0.001$ & \\
\hline $3 v 6$ & $p<0.001$ & & $p<0.001$ & $p<0.001$ & $p<0.001$ \\
\hline $4 v 5$ & $p<0.001$ & & $p<0.001$ & $p<0.001$ & $p<0.001$ \\
\hline $4 v 6$ & $p<0.001$ & & $p<0.001$ & $p<0.001$ & $p<0.001$ \\
\hline $5 v 6$ & & & $p<0.001$ & & $p<0.001$ \\
\hline *All Post-Hoc via D & t's T3, Except & idity (oqualv? & : Bonferroni) & & \\
\hline * Draft Rates ranked & to analysis $f$ & 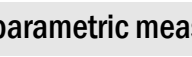 & & & \\
\hline
\end{tabular}


Table 4. Task 01 Bldg 002 by Zone

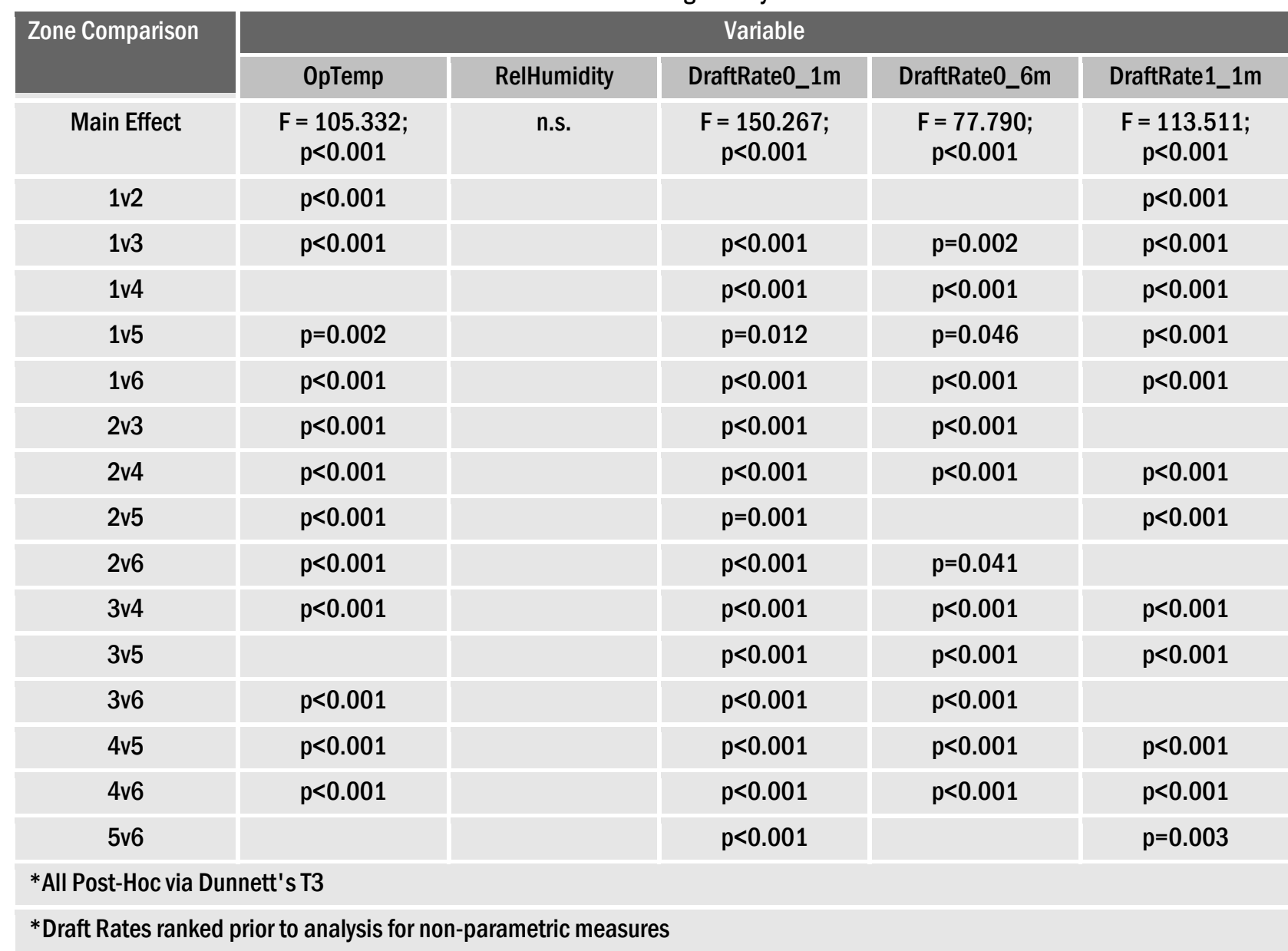


Table 5. Task 01 Bldg 003 by Zone

\begin{tabular}{|c|c|c|c|c|c|}
\hline \multirow[t]{2}{*}{ Zone Comparison } & \multicolumn{5}{|c|}{ Variable } \\
\hline & OpTemp & RelHumidity & DraftRate0_1m & DraftRate0_6m & DraftRate1_1m \\
\hline Main Effect & $\begin{array}{c}F=451.604 \\
p<0.001\end{array}$ & n.s. & $\begin{array}{c}F=98.685 \\
p<0.001\end{array}$ & $\begin{array}{c}F=123.225 \\
p<0.001\end{array}$ & $\begin{array}{c}F=84.119 \\
p<0.001\end{array}$ \\
\hline 1v2 & $p<0.001$ & & $p<0.001$ & $p<0.001$ & $p<0.001$ \\
\hline $1 v 3$ & $p<0.001$ & & $p<0.001$ & $p<0.001$ & $p<0.001$ \\
\hline $1 v 4$ & $p<0.001$ & & & $p<0.001$ & $p<0.001$ \\
\hline $1 v 5$ & $p<0.001$ & & $p=0.015$ & $p<0.001$ & $p<0.001$ \\
\hline $1 v 6$ & $p<0.001$ & & $p<0.001$ & $p<0.001$ & $p<0.001$ \\
\hline $2 v 3$ & $p<0.001$ & & $p<0.001$ & $p<0.001$ & $p<0.001$ \\
\hline $2 v 4$ & & & $p=0.007$ & $p=0.010$ & \\
\hline $2 v 5$ & $p<0.001$ & & & $p<0.001$ & $p<0.001$ \\
\hline $2 v 6$ & $p<0.001$ & & $p<0.001$ & $p<0.001$ & $p<0.001$ \\
\hline $3 v 4$ & $p<0.001$ & & $p<0.001$ & $p<0.001$ & $p<0.001$ \\
\hline $3 v 5$ & $p<0.001$ & & $p<0.001$ & $p<0.001$ & $p<0.001$ \\
\hline $3 v 6$ & $p<0.001$ & & & & $p<0.001$ \\
\hline $4 v 5$ & $p<0.001$ & & & & $p<0.001$ \\
\hline $4 v 6$ & $p<0.001$ & & $p<0.001$ & $p<0.001$ & $p=0.001$ \\
\hline $5 v 6$ & $p<0.001$ & & $p<0.001$ & $p<0.001$ & \\
\hline \multicolumn{6}{|c|}{ *All Post-Hoc via Dunnett's T3 } \\
\hline
\end{tabular}


Table 6. Task 01 Bldg 004 by Zone

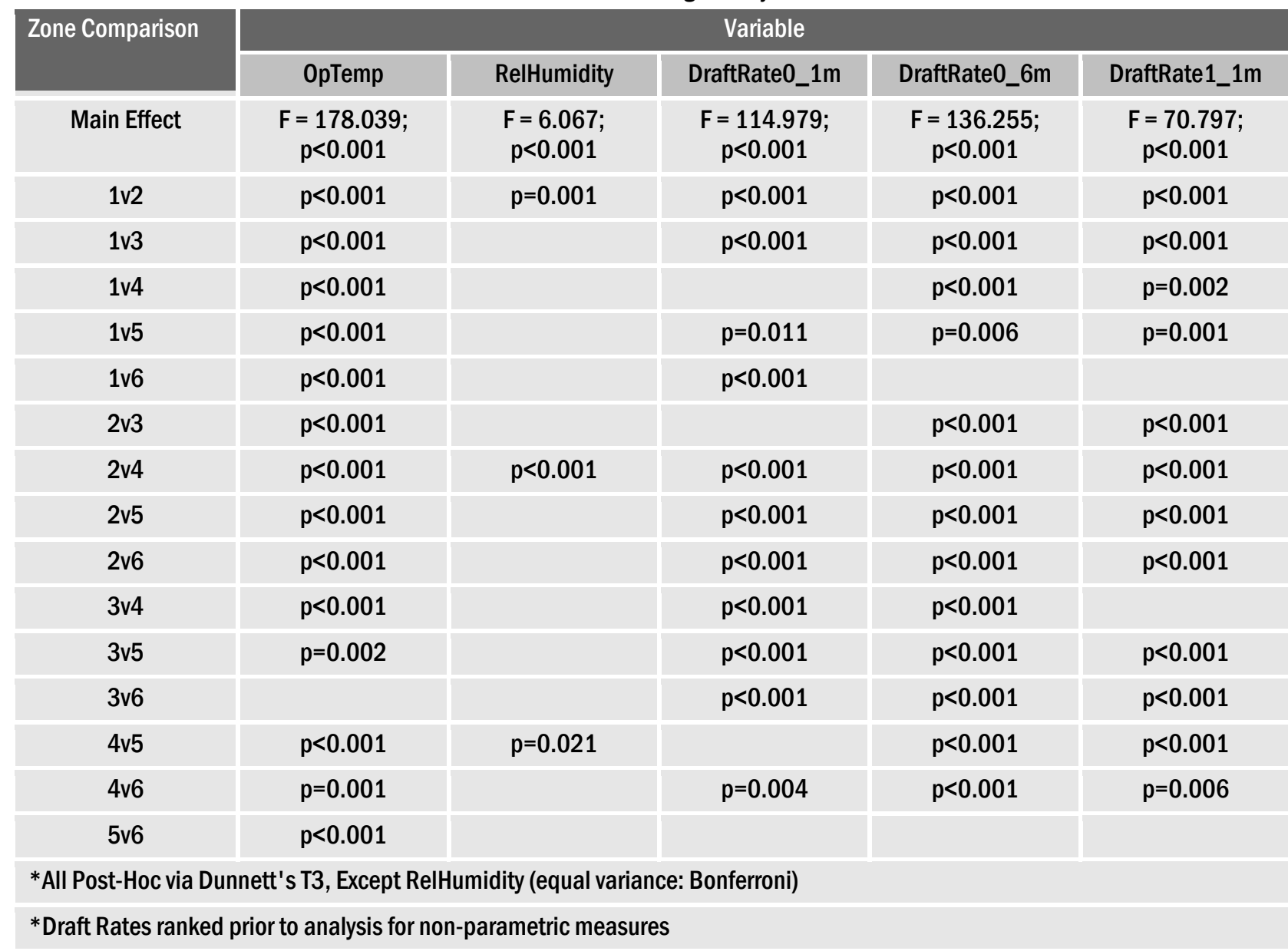


Table 7. Task 01 Bldg 005 by Zone

\begin{tabular}{|c|c|c|c|c|c|}
\hline \multirow[t]{2}{*}{ Zone Comparison } & \multicolumn{5}{|c|}{ Variable } \\
\hline & OpTemp & RelHumidity & DraftRate0_1m & DraftRate0_6m & DraftRate1_1m \\
\hline Main Effect & $\begin{array}{c}F=120.036 \\
p<0.001\end{array}$ & $\begin{array}{c}F=180.962 \\
p<0.001\end{array}$ & $\begin{array}{c}F=112.362 \\
p<0.001\end{array}$ & $\begin{array}{c}F=114.662 \\
p<0.001\end{array}$ & $\begin{array}{c}F=66.338 \\
p<0.001\end{array}$ \\
\hline $1 v 2$ & $p<0.001$ & $p<0.001$ & $p<0.001$ & $p<0.001$ & $p=0.012$ \\
\hline $1 v 3$ & $p<0.001$ & $p<0.001$ & $p<0.001$ & $p<0.001$ & $p=0.006$ \\
\hline $1 v 4$ & $p<0.001$ & $p<0.001$ & $p<0.001$ & & $p=0.004$ \\
\hline $1 v 5$ & $p<0.001$ & $p<0.001$ & $p<0.001$ & $p<0.001$ & $p<0.001$ \\
\hline $1 v 6$ & $p<0.001$ & $p<0.001$ & $p<0.001$ & $p<0.001$ & $p<0.001$ \\
\hline $2 v 3$ & $p<0.001$ & & & $p<0.001$ & $p<0.001$ \\
\hline $2 v 4$ & $p<0.001$ & $p<0.001$ & & & \\
\hline $2 v 5$ & $p<0.001$ & $p<0.001$ & $p<0.001$ & $p<0.001$ & $p<0.001$ \\
\hline $2 v 6$ & $p<0.001$ & $p<0.001$ & $p<0.001$ & $p<0.001$ & $p<0.001$ \\
\hline $3 v 4$ & $p<0.001$ & $p<0.001$ & & $p<0.001$ & $p<0.001$ \\
\hline $3 v 5$ & $p<0.001$ & $p<0.001$ & $p<0.001$ & $p<0.001$ & $p<0.001$ \\
\hline $3 v 6$ & $p<0.001$ & $p<0.001$ & $p<0.001$ & & $p=0.031$ \\
\hline $4 v 5$ & $p<0.001$ & $p<0.001$ & $p<0.001$ & $p<0.001$ & $p<0.001$ \\
\hline $4 v 6$ & & & $p<0.001$ & $p<0.001$ & $p<0.001$ \\
\hline $5 v 6$ & $p<0.001$ & $p=0.001$ & $p<0.001$ & $p<0.001$ & $p<0.001$ \\
\hline *All Post-Hoc via D & 's T3 & & & & \\
\hline
\end{tabular}


Table 8. Task 01 Bldg 006 by Zone

\begin{tabular}{|c|c|c|c|c|c|}
\hline \multirow{2}{*}{ Zone Comparison } & \multicolumn{5}{|c|}{ Variable } \\
\hline & OpTemp & RelHumidity & DraftRate0_1m & DraftRate0_6m & DraftRate1_1m \\
\hline Main Effect & $\begin{array}{c}F=18.646 \\
p<0.001\end{array}$ & $\begin{array}{c}F=11.340 \\
p<0.001\end{array}$ & $\begin{array}{c}F=255.748 \\
p<0.001\end{array}$ & $\begin{array}{c}F=317.352 \\
p<0.001\end{array}$ & $\begin{array}{c}F=268.340 \\
p<0.001\end{array}$ \\
\hline 1v2 & $p<0.001$ & & $p<0.001$ & $p<0.001$ & $p=0.001$ \\
\hline 1v3 & $p=0.020$ & $p<0.001$ & $p=0.001$ & $p<0.001$ & $p<0.001$ \\
\hline $1 v 4$ & $p<0.001$ & & $p<0.001$ & $p<0.001$ & $p<0.001$ \\
\hline $1 v 5$ & & & $p<0.001$ & $p=0.025$ & $p<0.001$ \\
\hline $1 v 6$ & & $p=0.002$ & $p=0.009$ & $p<0.001$ & $p<0.001$ \\
\hline $2 v 3$ & $p<0.001$ & $p<0.001$ & $p<0.001$ & $p<0.001$ & $p<0.001$ \\
\hline $2 v 4$ & $p=0.019$ & & & $p<0.001$ & $p<0.001$ \\
\hline $2 v 5$ & $p<0.001$ & & $p<0.001$ & $p<0.001$ & \\
\hline $2 v 6$ & & $p=0.006$ & $p<0.001$ & $p<0.001$ & $p<0.001$ \\
\hline $3 v 4$ & $p<0.001$ & $p<0.001$ & $p<0.001$ & $p<0.001$ & \\
\hline $3 v 5$ & $p<0.001$ & $p=0.016$ & $p<0.001$ & $p<0.001$ & $p<0.001$ \\
\hline $3 v 6$ & & & & $p<0.001$ & $p<0.001$ \\
\hline $4 v 5$ & $p<0.001$ & & $p<0.001$ & $p<0.001$ & $p<0.001$ \\
\hline $4 v 6$ & & $p=0.039$ & $p<0.001$ & $p<0.001$ & $p<0.001$ \\
\hline $5 v 6$ & & & $p<0.001$ & $p<0.001$ & $p<0.001$ \\
\hline *All Post-Hoc via Du & 's T3 & & & & \\
\hline
\end{tabular}


Table 9. Task 01 Bldg 007 by Zone

\begin{tabular}{|c|c|c|c|c|c|}
\hline \multirow{2}{*}{ Zone Comparison } & \multicolumn{5}{|c|}{ Variable } \\
\hline & OpTemp & RelHumidity & DraftRate0_1m & DraftRate0_6m & DraftRate1_1m \\
\hline Main Effect & $\begin{array}{c}F=54.496 \\
p<0.001\end{array}$ & $\begin{array}{c}F=14.160 \\
p<0.001\end{array}$ & $\begin{array}{c}F=115.061 \\
p<0.001\end{array}$ & $\begin{array}{c}F=120.424 \\
p<0.001\end{array}$ & $\begin{array}{c}F=129.094 \\
p<0.001\end{array}$ \\
\hline $1 v 2$ & $p<0.001$ & $p=0.031$ & $p<0.001$ & $p<0.001$ & $p<0.001$ \\
\hline $1 v 3$ & & $p<0.001$ & $p<0.001$ & $p<0.001$ & $p<0.001$ \\
\hline 1v4 & $p<0.001$ & & $p<0.001$ & $p<0.001$ & $p<0.001$ \\
\hline $1 v 5$ & & & $p<0.001$ & $p<0.001$ & $p<0.001$ \\
\hline $1 v 6$ & $p<0.001$ & $p=0.007$ & $p<0.001$ & $p<0.001$ & $p<0.001$ \\
\hline $2 v 3$ & $p<0.001$ & $p<0.001$ & & $p<0.001$ & $p=0.001$ \\
\hline $2 v 4$ & $p=0.014$ & $p=0.006$ & $p<0.001$ & $p<0.001$ & $p<0.001$ \\
\hline $2 v 5$ & $p=0.001$ & $p=0.001$ & $p<0.001$ & & $p<0.001$ \\
\hline $2 v 6$ & & $p<0.001$ & & $p<0.001$ & $p<0.001$ \\
\hline $3 v 4$ & $p<0.001$ & & $p<0.001$ & $p<0.001$ & $p<0.001$ \\
\hline $3 v 5$ & & $p=0.031$ & $p<0.001$ & $p<0.001$ & $p<0.001$ \\
\hline $3 v 6$ & $p<0.001$ & & & & $p<0.001$ \\
\hline $4 v 5$ & $p<0.001$ & & & $p<0.001$ & \\
\hline $4 v 6$ & $p<0.001$ & & $p<0.001$ & $p<0.001$ & $p<0.001$ \\
\hline $5 v 6$ & $p<0.001$ & & $p<0.001$ & $p<0.001$ & $p<0.001$ \\
\hline *All Post-Hoc via D & t's T3 & & & & \\
\hline
\end{tabular}


Table 10. Task 01 Bldg 008 by Zone

\begin{tabular}{|c|c|c|c|c|c|}
\hline \multirow{2}{*}{ Zone Comparison } & \multicolumn{5}{|c|}{ Variable } \\
\hline & OpTemp & RelHumidity & DraftRate0_1m & DraftRate0_6m & DraftRate1_1m \\
\hline Main Effect & $\begin{array}{c}F=339.069 \\
p<0.001\end{array}$ & $\begin{array}{c}F=6.090 \\
p<0.001\end{array}$ & $\begin{array}{c}F=79.227 \\
p<0.001\end{array}$ & $\begin{array}{c}F=65.801 \\
p<0.001\end{array}$ & $\begin{array}{c}F=80.260 \\
p<0.001\end{array}$ \\
\hline 1v2 & $p<0.001$ & & & $p<0.001$ & \\
\hline $1 v 3$ & & & excluded & & Excluded \\
\hline $1 v 4$ & & & $p<0.001$ & $p<0.001$ & $p<0.001$ \\
\hline $1 v 5$ & $p<0.001$ & $p=0.023$ & $p<0.001$ & & \\
\hline $1 v 6$ & $p<0.001$ & & $p<0.001$ & $p<0.001$ & $p<0.001$ \\
\hline $2 v 3$ & $p<0.001$ & & excluded & $p<0.001$ & Excluded \\
\hline $2 v 4$ & $p<0.001$ & & $p<0.001$ & $p=0.006$ & $p<0.001$ \\
\hline 2v5 & $p<0.001$ & $p<0.001$ & $p<0.001$ & $p<0.001$ & \\
\hline $2 v 6$ & $p<0.001$ & $p=0.011$ & $p<0.001$ & $p<0.001$ & $p<0.001$ \\
\hline $3 v 4$ & & & excluded & $p<0.001$ & Excluded \\
\hline $3 v 5$ & $p<0.001$ & $p=0.008$ & excluded & & Excluded \\
\hline $3 v 6$ & $p<0.001$ & & excluded & $p<0.001$ & excluded \\
\hline $4 v 5$ & $p<0.001$ & $p=0.009$ & $p<0.001$ & $p<0.001$ & $p<0.001$ \\
\hline $4 v 6$ & $p<0.001$ & & & & \\
\hline $5 v 6$ & $p<0.001$ & & $p<0.001$ & $p<0.001$ & $p<0.001$ \\
\hline
\end{tabular}


Table 11. Task 01 Bldg 009 by Zone

\begin{tabular}{|c|c|c|c|c|c|}
\hline \multirow{2}{*}{ Zone Comparison } & \multicolumn{5}{|c|}{ Variable } \\
\hline & OpTemp & RelHumidity & DraftRate0_1m & DraftRate0_6m & DraftRate1_1m \\
\hline Main Effect & $\begin{array}{c}F=19.530 \\
p<0.001\end{array}$ & n.s. & $\begin{array}{c}F=166.519 \\
p<0.001\end{array}$ & $\begin{array}{c}F=100.600 \\
p<0.001\end{array}$ & $\begin{array}{c}F=57.698 \\
p<0.001\end{array}$ \\
\hline 1v2 & $p<0.001$ & & $p<0.001$ & $p<0.001$ & $p<0.001$ \\
\hline $1 v 3$ & & & $p<0.001$ & $p<0.001$ & excluded \\
\hline $1 v 4$ & & & & $p<0.001$ & $p<0.001$ \\
\hline $1 v 5$ & & & & $p<0.001$ & $p<0.001$ \\
\hline $1 v 6$ & $p=0.001$ & & $p<0.001$ & $p<0.001$ & $p<0.001$ \\
\hline $2 v 3$ & $p<0.001$ & & $p<0.001$ & $p<0.001$ & excluded \\
\hline $2 v 4$ & $p<0.001$ & & $p<0.001$ & $p<0.001$ & $p<0.001$ \\
\hline $2 v 5$ & $p<0.001$ & & $p<0.001$ & $p<0.001$ & $p<0.001$ \\
\hline $2 v 6$ & & & & $p=0.003$ & $p=0.007$ \\
\hline $3 v 4$ & & & $p<0.001$ & & excluded \\
\hline $3 v 5$ & & & $p<0.001$ & & excluded \\
\hline $3 v 6$ & $p=0.001$ & & $p<0.001$ & $p<0.001$ & excluded \\
\hline \multicolumn{6}{|l|}{$4 v 5$} \\
\hline $4 v 6$ & $p<0.001$ & & $p<0.001$ & $p<0.001$ & $p=0.007$ \\
\hline $5 v 6$ & $p=0.002$ & & $p<0.001$ & $p<0.001$ & $p<0.001$ \\
\hline \multicolumn{6}{|c|}{ *All Post-Hoc via Dunnett's T3 } \\
\hline
\end{tabular}


Table 12. Task 01 Bldg 010 by Zone

\begin{tabular}{|c|c|c|c|c|c|}
\hline \multirow{2}{*}{$\begin{array}{c}\text { Zone } \\
\text { Comparison }\end{array}$} & \multicolumn{5}{|c|}{ Variable } \\
\hline & OpTemp & RelHumidity & DraftRate0_1m & DraftRate0_6m & DraftRate1_1m \\
\hline Main Effect & $\begin{array}{c}F=120.333 \\
p<0.001\end{array}$ & $\begin{array}{c}F=3.939 \\
p=0.002\end{array}$ & $\begin{array}{c}F=251.987 \\
p<0.001\end{array}$ & $\begin{array}{c}F=238.407 \\
p<0.001\end{array}$ & $\begin{array}{c}F=163.735 \\
p<0.001\end{array}$ \\
\hline $1 \mathrm{v} 2$ & $p<0.001$ & & & $p=0.003$ & \\
\hline 1v3 & $p<0.001$ & $p=0.009$ & $p<0.001$ & $p<0.001$ & excluded \\
\hline $1 v 4$ & $p<0.001$ & $p=0.004$ & $p<0.001$ & $p<0.001$ & $p<0.001$ \\
\hline $1 v 5$ & $p<0.001$ & & $p<0.001$ & $p<0.001$ & $p<0.001$ \\
\hline $1 v 6$ & & $p=0.018$ & $p<0.001$ & $p<0.001$ & $p<0.001$ \\
\hline $2 v 3$ & $p<0.001$ & & $p<0.001$ & $p<0.001$ & excluded \\
\hline $2 v 4$ & $p<0.001$ & & $p<0.001$ & $p<0.001$ & $p<0.001$ \\
\hline $2 v 5$ & $p<0.001$ & & $p<0.001$ & $p<0.001$ & $p<0.001$ \\
\hline $2 v 6$ & $p=0.018$ & & $p<0.001$ & $p<0.001$ & $p<0.001$ \\
\hline $3 v 4$ & $p=0.008$ & & $p<0.001$ & $p<0.001$ & excluded \\
\hline $3 v 5$ & $p<0.001$ & & $p<0.001$ & $p<0.001$ & excluded \\
\hline $3 v 6$ & $p<0.001$ & & $p<0.001$ & $p=0.017$ & excluded \\
\hline $4 v 5$ & & & $p<0.001$ & $p<0.001$ & $p<0.001$ \\
\hline $4 v 6$ & $p<0.001$ & & $p=0.003$ & & \\
\hline $5 v 6$ & $p<0.001$ & & $p<0.001$ & $p<0.001$ & $p<0.001$ \\
\hline${ }^{*}$ All Post-Hoc v & Inett's T3, Exce & Humidity (equa & nce: Bonferroni) & & \\
\hline
\end{tabular}


Table 13. Task 13 Bldg S01 by Zone

\begin{tabular}{|c|c|c|c|c|c|}
\hline \multirow{2}{*}{ Zone Comparison } & \multicolumn{5}{|c|}{ Variable } \\
\hline & OpTemp & RelHumidity & DraftRate0_1m & DraftRate0_6m & DraftRate1_1m \\
\hline Main Effect & $\begin{array}{c}F=144.489 \\
p<0.001\end{array}$ & n.s. & $\begin{array}{c}F=32.315 \\
p<0.001\end{array}$ & $\begin{array}{c}F=127.300 \\
p<0.001\end{array}$ & $\begin{array}{c}F=123.314 \\
p<0.001\end{array}$ \\
\hline $1 v 2$ & $p<0.001$ & & & $p<0.001$ & $p<0.001$ \\
\hline 1v3 & $p<0.001$ & & $p=0.030$ & $p<0.001$ & $p<0.001$ \\
\hline $1 v 4$ & $p<0.001$ & & $p<0.001$ & $p<0.001$ & $p<0.001$ \\
\hline $1 v 5$ & $p<0.001$ & & & & \\
\hline \multicolumn{6}{|l|}{$1 v 6$} \\
\hline $2 v 3$ & $p<0.001$ & & & & $p<0.001$ \\
\hline $2 v 4$ & $p<0.001$ & & $p<0.001$ & $p<0.001$ & $p<0.001$ \\
\hline $2 v 5$ & $p<0.001$ & & & $p<0.001$ & $p<0.001$ \\
\hline $2 v 6$ & $p<0.001$ & & $p=0.022$ & $p<0.001$ & $p<0.001$ \\
\hline $3 v 4$ & $p<0.001$ & & $p<0.001$ & $p<0.001$ & $p<0.001$ \\
\hline $3 v 5$ & $p<0.001$ & & & $p<0.001$ & $p<0.001$ \\
\hline $3 v 6$ & $p<0.001$ & & $p=0.009$ & $p<0.001$ & $p<0.001$ \\
\hline $4 v 5$ & $p=0.019$ & & $p<0.001$ & $p<0.001$ & $p<0.001$ \\
\hline $4 v 6$ & & & $p<0.001$ & $p<0.001$ & $p<0.001$ \\
\hline $5 v 6$ & $p<0.001$ & & & & \\
\hline \multicolumn{6}{|c|}{ *All Post-Hoc via Dunnett's T3 } \\
\hline \multicolumn{6}{|c|}{ * Draft Rates ranked prior to analysis for non-parametric measures } \\
\hline
\end{tabular}


Table 14. Task 13 Bldg S02 by Zone

\begin{tabular}{|c|c|c|c|c|c|}
\hline \multirow{2}{*}{$\begin{array}{c}\text { Zone } \\
\text { Comparison }\end{array}$} & \multicolumn{5}{|c|}{ Variable } \\
\hline & OpTemp & RelHumidity & DraftRate0_1m & DraftRate0_6m & DraftRate1_1m \\
\hline Main Effect & $\begin{array}{c}F=322.011 \\
p<0.001\end{array}$ & $\begin{array}{c}F=10.632 \\
p<0.001\end{array}$ & $\begin{array}{c}F=107.348 \\
p<0.001\end{array}$ & $\begin{array}{c}F=68.678 \\
p<0.001\end{array}$ & $\begin{array}{c}F=35.805 \\
p<0.001\end{array}$ \\
\hline $1 v 2$ & $p<0.001$ & $p<0.001$ & $p<0.001$ & $p<0.001$ & $p<0.001$ \\
\hline 1v3 & $p<0.001$ & & $p<0.001$ & $p<0.001$ & \\
\hline 1v4 & $p<0.001$ & $p=0.004$ & $p<0.001$ & & $p<0.001$ \\
\hline $1 v 5$ & $p<0.001$ & & $p<0.001$ & & $p<0.001$ \\
\hline $1 v 6$ & $p<0.001$ & $p<0.001$ & $p<0.001$ & $p<0.001$ & \\
\hline $2 v 3$ & $p<0.001$ & $p=0.002$ & & $p<0.001$ & $p=0.001$ \\
\hline 2v4 & $p<0.001$ & & $p<0.001$ & $p<0.001$ & $p<0.001$ \\
\hline $2 v 5$ & $p<0.001$ & $p=0.003$ & $p<0.001$ & $p<0.001$ & $p<0.001$ \\
\hline $2 v 6$ & $p<0.001$ & & $p<0.001$ & & $p<0.001$ \\
\hline $3 v 4$ & $p<0.001$ & & $p<0.001$ & $p=0.031$ & $p<0.001$ \\
\hline $3 v 5$ & $p<0.001$ & & $p<0.001$ & $p<0.001$ & $p<0.001$ \\
\hline $3 v 6$ & $p<0.001$ & $p=0.012$ & $p<0.001$ & $p<0.001$ & \\
\hline $4 v 5$ & $p<0.001$ & & $p=0.014$ & & \\
\hline $4 v 6$ & $p<0.001$ & & $p<0.001$ & $p<0.001$ & $p<0.001$ \\
\hline $5 v 6$ & $p<0.001$ & $p=0.017$ & $p<0.001$ & $p<0.001$ & $p<0.001$ \\
\hline \multicolumn{6}{|c|}{ *All Post-Hoc via Dunnett's T3 } \\
\hline
\end{tabular}


Table 15. Task 13 Bldg S03 by Zone

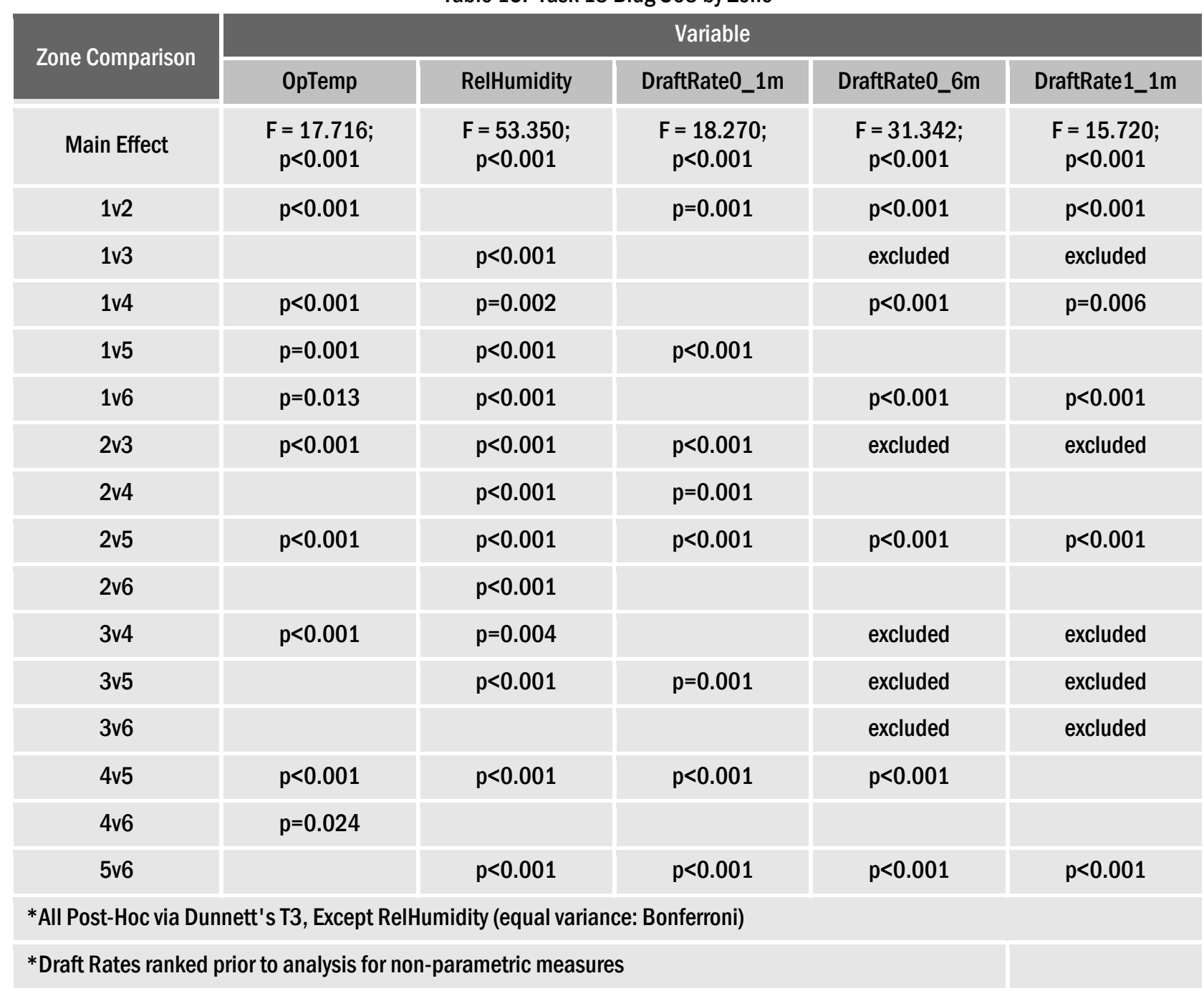


Table 16. Task 13 Bldg S04 by Zone

\begin{tabular}{|c|c|c|c|c|c|}
\hline \multirow{2}{*}{$\begin{array}{c}\text { Zone } \\
\text { Comparison }\end{array}$} & \multicolumn{5}{|c|}{ Variable } \\
\hline & OpTemp & RelHumidity & DraftRate0_1m & DraftRate0_6m & DraftRate1_1m \\
\hline Main Effect & $\begin{array}{c}F=32.248 \\
p<0.001\end{array}$ & n.s. & $\begin{array}{c}F=24.217 \\
p<0.001\end{array}$ & $\begin{array}{c}F=15.077 \\
p<0.001\end{array}$ & $\begin{array}{c}F=24.627 \\
p<0.001\end{array}$ \\
\hline \multicolumn{6}{|l|}{$1 v 2$} \\
\hline $1 v 3$ & $p<0.001$ & & & $p=0.002$ & $p<0.001$ \\
\hline $1 v 4$ & $p=0.001$ & & $p<0.001$ & & \\
\hline $1 v 5$ & $p<0.001$ & & & $p=0.005$ & $p<0.001$ \\
\hline $1 v 6$ & $p<0.001$ & & $p<0.001$ & & $p<0.001$ \\
\hline $2 v 3$ & & & & $p=0.018$ & $p<0.001$ \\
\hline $2 v 4$ & $p=0.014$ & & $p<0.001$ & & \\
\hline $2 v 5$ & $p<0.001$ & & & & $p<0.001$ \\
\hline $2 v 6$ & & & $p=0.004$ & & $p=0.001$ \\
\hline $3 v 4$ & $p<0.001$ & & $p<0.001$ & $p<0.001$ & $p<0.001$ \\
\hline $3 v 5$ & $p<0.001$ & & & & \\
\hline $3 v 6$ & & & $p<0.001$ & $p<0.001$ & \\
\hline $4 v 5$ & $p<0.001$ & & $p<0.001$ & $p<0.001$ & $p<0.001$ \\
\hline $4 v 6$ & $p<0.001$ & & $p=0.001$ & & $p<0.001$ \\
\hline $5 v 6$ & $p<0.001$ & & $p=0.007$ & $p<0.001$ & \\
\hline \multicolumn{6}{|c|}{ *All Post-Hoc via Dunnett's T3 } \\
\hline *Draft Rates ra & ior to analysi & -narametric & & & \\
\hline
\end{tabular}


Table 17. Task 13 Bldg S05 by Zone

\begin{tabular}{|c|c|c|c|c|c|}
\hline \multirow{2}{*}{ Zone Comparison } & \multicolumn{5}{|c|}{ Variable } \\
\hline & OpTemp & RelHumidity & DraftRate0_1m & DraftRate0_6m & DraftRate1_1m \\
\hline Main Effect & $\begin{array}{c}F=776.266 \\
p<0.001\end{array}$ & $\begin{array}{c}F=5.412 \\
p<0.001\end{array}$ & $\begin{array}{c}F=62.757 \\
p<0.001\end{array}$ & $\begin{array}{c}F=63.178 \\
p<0.001\end{array}$ & $\begin{array}{c}F=78.850 \\
p<0.001\end{array}$ \\
\hline $1 v 2$ & & & & $p<0.001$ & \\
\hline $1 v 3$ & $p<0.001$ & $p<0.001$ & & & $p<0.001$ \\
\hline $1 v 4$ & $p<0.001$ & & $p<0.001$ & $p<0.001$ & $p<0.001$ \\
\hline $1 v 5$ & $p<0.001$ & & $p<0.001$ & & $p<0.001$ \\
\hline $1 v 6$ & $p<0.001$ & & $p<0.001$ & $p<0.001$ & \\
\hline $2 v 3$ & $p<0.001$ & $p<0.001$ & & $p<0.001$ & $p<0.001$ \\
\hline $2 v 4$ & $p<0.001$ & & $p<0.001$ & $p<0.001$ & $p<0.001$ \\
\hline $2 v 5$ & $p=0.001$ & & $p<0.001$ & $p<0.001$ & $p<0.001$ \\
\hline $2 v 6$ & $p<0.001$ & & $p<0.001$ & $p=0.003$ & \\
\hline $3 v 4$ & $p<0.001$ & $p=0.005$ & $p<0.001$ & $p<0.001$ & $p<0.001$ \\
\hline $3 v 5$ & $p<0.001$ & $p=0.005$ & $p<0.001$ & & \\
\hline $3 v 6$ & $p<0.001$ & & $p<0.001$ & $p<0.001$ & $p<0.001$ \\
\hline $4 v 5$ & $p<0.001$ & & $p<0.001$ & $p<0.001$ & $p<0.001$ \\
\hline $4 v 6$ & $p<0.001$ & & $p=0.033$ & $p<0.001$ & $p<0.001$ \\
\hline $5 v 6$ & $p<0.001$ & & & $p<0.001$ & $p<0.001$ \\
\hline All Post-Hoc via Du & 's T3, Except D & 1_1m (equa & Ice: Bonferroni) & & \\
\hline
\end{tabular}


Table 18. Task 13 Bldg S06 by Zone

\begin{tabular}{|c|c|c|c|c|c|}
\hline \multirow{2}{*}{$\begin{array}{c}\text { Zone } \\
\text { Comparison }\end{array}$} & \multicolumn{5}{|c|}{ Variable } \\
\hline & OpTemp & RelHumidity & DraftRate0_1m & DraftRate0_6m & DraftRate1_1m \\
\hline Main Effect & $\begin{array}{c}F=59.823 \\
p<0.001\end{array}$ & $\begin{array}{c}F=49.156 \\
p<0.001\end{array}$ & $\begin{array}{c}F=41.469 \\
p<0.001\end{array}$ & $\begin{array}{c}F=108.125 \\
p<0.001\end{array}$ & $\begin{array}{c}F=38.401 \\
p<0.001\end{array}$ \\
\hline $1 v 2$ & $p<0.001$ & & $p<0.001$ & $p=0.005$ & \\
\hline $1 \mathrm{v} 3$ & $p<0.001$ & & & $p<0.001$ & excluded \\
\hline $1 v 4$ & $p<0.001$ & $p<0.001$ & & $p<0.001$ & $p<0.001$ \\
\hline $1 v 5$ & $p<0.001$ & $p<0.001$ & & & $p<0.001$ \\
\hline $1 v 6$ & $p<0.001$ & $p<0.001$ & $p<0.001$ & $p<0.001$ & $p<0.001$ \\
\hline $2 v 3$ & $p<0.001$ & & $p<0.001$ & $p<0.001$ & excluded \\
\hline $2 v 4$ & & $p<0.001$ & $p<0.001$ & $p<0.001$ & $p<0.001$ \\
\hline $2 \mathrm{v} 5$ & & $p=0.001$ & $p<0.001$ & & \\
\hline $2 v 6$ & $p<0.001$ & $p<0.001$ & $p<0.001$ & $p<0.001$ & $p<0.001$ \\
\hline $3 v 4$ & $p<0.001$ & $p<0.001$ & & $p=0.002$ & excluded \\
\hline $3 v 5$ & & $p<0.001$ & & $p<0.001$ & excluded \\
\hline $3 v 6$ & $p<0.001$ & $p<0.001$ & $p<0.001$ & $p<0.001$ & excluded \\
\hline $4 v 5$ & & $p<0.001$ & & $p<0.001$ & \\
\hline $4 v 6$ & $p<0.001$ & $p=0.042$ & $p<0.001$ & $p<0.001$ & $p<0.001$ \\
\hline $5 v 6$ & $p=0.001$ & $p<0.001$ & $p<0.001$ & $p<0.001$ & $p<0.001$ \\
\hline \multicolumn{6}{|c|}{ *All Post-Hoc via Dunnett's T3 } \\
\hline
\end{tabular}


Table 19. . Task 13 Bldg S07 by Zone

\begin{tabular}{|c|c|c|c|c|c|}
\hline \multirow{2}{*}{ Zone Comparison } & \multicolumn{5}{|c|}{ Variable } \\
\hline & OpTemp & RelHumidity & DraftRate0_1m & DraftRate0_6m & DraftRate1_1m \\
\hline Main Effect & $\begin{array}{c}F=106.830 \\
p<0.001\end{array}$ & $\begin{array}{c}F=29.965 \\
p<0.001\end{array}$ & $\begin{array}{c}F=69.810 \\
p<0.001\end{array}$ & $\begin{array}{c}F=24.654 \\
p<0.001\end{array}$ & $\begin{array}{c}F=13.894 \\
p<0.001\end{array}$ \\
\hline $1 v 2$ & $p<0.001$ & $p<0.001$ & $p<0.001$ & $p=0.001$ & $p<0.001$ \\
\hline $1 v 3$ & $p<0.001$ & $p<0.001$ & excluded & excluded & excluded \\
\hline $1 v 4$ & $p<0.001$ & $p<0.001$ & $p=0.004$ & & \\
\hline $1 v 5$ & $p<0.001$ & $p<0.001$ & $p<0.001$ & $p<0.001$ & $p=0.002$ \\
\hline $1 v 6$ & $p<0.001$ & $p<0.001$ & $p<0.001$ & $p<0.001$ & $p<0.001$ \\
\hline 2v3 & $p=0.001$ & & excluded & excluded & excluded \\
\hline $2 v 4$ & $p<0.001$ & $p<0.001$ & $p<0.001$ & $p<0.001$ & \\
\hline $2 v 5$ & $p<0.001$ & & $p<0.001$ & $p=0.005$ & \\
\hline $2 v 6$ & $p<0.001$ & $p=0.022$ & & $p<0.001$ & \\
\hline $3 v 4$ & $p<0.001$ & $p<0.001$ & excluded & excluded & excluded \\
\hline $3 v 5$ & $p<0.001$ & $p=0.006$ & excluded & excluded & excluded \\
\hline $3 v 6$ & $p<0.001$ & $p=0.001$ & excluded & excluded & excluded \\
\hline $4 v 5$ & $p<0.001$ & & $p<0.001$ & $p=0.025$ & \\
\hline $4 v 6$ & $p=0.002$ & & & & $p=0.014$ \\
\hline $5 v 6$ & & & $p<0.001$ & & $p=0.044$ \\
\hline *All Post-Hoc via Du & 's T3 & & & & \\
\hline
\end{tabular}


Table 20. Task 13 Bldg S08 by Zone

\begin{tabular}{|c|c|c|c|c|c|}
\hline \multirow{2}{*}{$\begin{array}{c}\text { Zone } \\
\text { Comparison }\end{array}$} & \multicolumn{5}{|c|}{ Variable } \\
\hline & OpTemp & RelHumidity & DraftRate0_1m & DraftRate0_6m & DraftRate1_1m \\
\hline Main Effect & $\begin{array}{c}F=15.197 \\
p<0.001\end{array}$ & $\begin{array}{c}F=2.817 \\
p=0.017\end{array}$ & $\begin{array}{c}F=69.114 \\
p<0.001\end{array}$ & $\begin{array}{c}F=26.845 \\
p<0.001\end{array}$ & $\begin{array}{c}F=50.091 \\
p<0.001\end{array}$ \\
\hline $1 v 2$ & $p=0.013$ & & $p=0.033$ & & \\
\hline $1 v 3$ & $p<0.001$ & $p=0.013$ & $p<0.001$ & $p<0.001$ & excluded \\
\hline $1 v 4$ & & & & $p=0.001$ & $p<0.001$ \\
\hline $1 v 5$ & $p=0.036$ & $p=0.029$ & $p<0.001$ & $p=0.003$ & \\
\hline $1 v 6$ & & & $p=0.011$ & $p<0.001$ & $p<0.001$ \\
\hline $2 v 3$ & $p<0.001$ & & $p<0.001$ & $p=0.004$ & excluded \\
\hline $2 v 4$ & $p<0.001$ & & & & $p<0.001$ \\
\hline $2 v 5$ & $p<0.001$ & & $p<0.001$ & & \\
\hline $2 v 6$ & $p=0.020$ & & & $p<0.001$ & $p<0.001$ \\
\hline $3 v 4$ & $p=0.015$ & & $p<0.001$ & $p=0.001$ & excluded \\
\hline $3 v 5$ & & & $p<0.001$ & $p=0.015$ & excluded \\
\hline $3 v 6$ & $p=0.001$ & & $p<0.001$ & $p=0.002$ & excluded \\
\hline $4 v 5$ & & & $p<0.001$ & & $p<0.001$ \\
\hline $4 v 6$ & & & & $p<0.001$ & $p=0.001$ \\
\hline $5 v 6$ & & & $p<0.001$ & $p<0.001$ & $p<0.001$ \\
\hline & *All Post-ト & Dunnett's T3, & RelHumidity (equ & iance: Bonferroni) & \\
\hline
\end{tabular}


Table 21. Task 13 Bldg S09 by Zone

\begin{tabular}{|c|c|c|c|c|c|}
\hline \multirow{2}{*}{ Zone Comparison } & \multicolumn{5}{|c|}{ Variable } \\
\hline & OpTemp & RelHumidity & DraftRate0_1m & DraftRate0_6m & DraftRate1_1m \\
\hline Main Effect & $\begin{array}{c}F=12.948 \\
p<0.001\end{array}$ & $\begin{array}{c}F=14.452 \\
p<0.001\end{array}$ & $\begin{array}{c}F=31.488 \\
p<0.001\end{array}$ & $\begin{array}{c}F=33.871 \\
p<0.001\end{array}$ & $\begin{array}{c}F=44.322 \\
p<0.001\end{array}$ \\
\hline 1v2 & & & $p<0.001$ & $p=0.016$ & $p<0.001$ \\
\hline 1v3 & excluded & $p=0.002$ & excluded & $p<0.001$ & excluded \\
\hline $1 v 4$ & $p<0.001$ & $p<0.001$ & & & $p=0.009$ \\
\hline $1 v 5$ & $p=0.010$ & $p<0.001$ & $p<0.001$ & $p<0.001$ & $p<0.001$ \\
\hline $1 v 6$ & $p=0.001$ & $p<0.001$ & $p<0.001$ & $p<0.001$ & $p<0.001$ \\
\hline 2v3 & excluded & $p=0.001$ & excluded & & excluded \\
\hline $2 v 4$ & $p<0.001$ & $p<0.001$ & $p<0.001$ & & \\
\hline $2 v 5$ & $p=0.003$ & $p<0.001$ & $p<0.001$ & $p<0.001$ & $p<0.001$ \\
\hline $2 v 6$ & $p<0.001$ & $p<0.001$ & & & \\
\hline $3 v 4$ & excluded & & excluded & $p<0.001$ & excluded \\
\hline $3 v 5$ & excluded & & excluded & $p<0.001$ & excluded \\
\hline $3 v 6$ & excluded & & excluded & & excluded \\
\hline $4 v 5$ & & & $p<0.001$ & $p<0.001$ & $p<0.001$ \\
\hline $4 v 6$ & & & $p<0.001$ & $p=0.001$ & \\
\hline $5 v 6$ & & & $p<0.001$ & $p<0.001$ & $p<0.001$ \\
\hline *All Post-Hoc via Du & s T3 & & & & \\
\hline
\end{tabular}


Table 22. Task 13 Bldg S10 by Zone

\begin{tabular}{|c|c|c|c|c|c|}
\hline \multirow{2}{*}{$\begin{array}{c}\text { Zone } \\
\text { Comparison }\end{array}$} & \multicolumn{5}{|c|}{ Variable } \\
\hline & OpTemp & RelHumidity & DraftRate0_1m & DraftRate0_6m & DraftRate1_1m \\
\hline Main Effect & $\begin{array}{c}F=48.496 \\
p<0.001\end{array}$ & $\begin{array}{c}F=10.119 \\
p<0.001\end{array}$ & $\begin{array}{c}F=81.085 \\
p<0.001\end{array}$ & $\begin{array}{c}F=13.788 \\
p<0.001\end{array}$ & $\begin{array}{c}F=10.859 \\
p<0.001\end{array}$ \\
\hline $1 \mathrm{v} 2$ & & & & $p=0.006$ & \\
\hline 1v3 & & & & & $p=0.006$ \\
\hline $1 v 4$ & $p=0.003$ & & $p<0.001$ & & \\
\hline $1 v 5$ & $p<0.001$ & $p=0.003$ & $p<0.001$ & $p=0.011$ & \\
\hline $1 v 6$ & $p<0.001$ & $p=0.001$ & $p<0.001$ & $p<0.001$ & \\
\hline $2 v 3$ & $p=0.001$ & & $p<0.001$ & $p=0.001$ & $p<0.001$ \\
\hline $2 v 4$ & $p<0.001$ & & $p<0.001$ & & \\
\hline $2 v 5$ & $p<0.001$ & $p<0.001$ & $p<0.001$ & & \\
\hline $2 v 6$ & $p<0.001$ & $p<0.001$ & $p<0.001$ & & \\
\hline $3 v 4$ & & & & $p=0.046$ & $p=0.004$ \\
\hline $3 v 5$ & $p<0.001$ & $p=0.004$ & $p<0.001$ & $p=0.003$ & $p=0.004$ \\
\hline $3 v 6$ & $p<0.001$ & $p=0.001$ & $p=0.004$ & $p<0.001$ & $p<0.001$ \\
\hline $4 v 5$ & $p<0.001$ & $p=0.006$ & $p<0.001$ & & \\
\hline $4 v 6$ & $p<0.001$ & $p=0.001$ & & $p<0.001$ & \\
\hline $5 v 6$ & & & $p<0.001$ & $p=0.007$ & \\
\hline${ }^{*}$ All Post-Hoc v & nett's T3, Exc & fumidity (eque & nce: Bonferroni) & & \\
\hline
\end{tabular}


Table 23. Task 06-06 Bldg HPB01 by Zone

\begin{tabular}{|c|c|c|c|c|c|}
\hline \multirow{2}{*}{ Zone Comparison } & \multicolumn{5}{|c|}{ Variable } \\
\hline & Temp & RelHumidity & DraftRate0_1m & DraftRate0_6m & DraftRate1_1m \\
\hline Main Effect & $\begin{array}{c}F=594.237 \\
p<0.001\end{array}$ & $\begin{array}{c}F=183.504 \\
p<0.001\end{array}$ & $\begin{array}{c}F=46.175 \\
p<0.001\end{array}$ & $\begin{array}{c}F=71.486 \\
p<0.001\end{array}$ & $\begin{array}{c}F=63.311 \\
p<0.001\end{array}$ \\
\hline 0v1 & $p<0.001$ & $p<0.001$ & excluded & excluded & excluded \\
\hline 0v2 & $p<0.001$ & $p<0.001$ & excluded & excluded & excluded \\
\hline 0v3 & $p<0.001$ & $p<0.001$ & excluded & excluded & excluded \\
\hline 0v4 & $p<0.001$ & $p<0.001$ & excluded & excluded & excluded \\
\hline 0v5 & $p<0.001$ & $p<0.001$ & excluded & excluded & excluded \\
\hline $0 v 6$ & $p<0.001$ & $p<0.001$ & excluded & excluded & excluded \\
\hline $1 v 2$ & $p<0.001$ & & $p<0.001$ & $p<0.001$ & $p<0.001$ \\
\hline $1 v 3$ & $p=0.024$ & & & & $p=0.024$ \\
\hline $1 v 4$ & $p<0.001$ & & & $p<0.001$ & $p<0.001$ \\
\hline $1 v 5$ & $p<0.001$ & & $p=0.009$ & $p=0.005$ & $p=0.001$ \\
\hline $1 v 6$ & & & $p<0.001$ & $p<0.001$ & $p<0.001$ \\
\hline $2 v 3$ & $p<0.001$ & & $p<0.001$ & $p<0.001$ & $p<0.001$ \\
\hline $2 v 4$ & $p<0.001$ & & $p<0.001$ & $p=0.044$ & \\
\hline $2 v 5$ & $p<0.001$ & & $p<0.001$ & $p<0.001$ & $p<0.001$ \\
\hline $2 v 6$ & $p<0.001$ & & $p=0.009$ & & \\
\hline $3 v 4$ & $p<0.001$ & & & $p<0.001$ & $p<0.001$ \\
\hline $3 v 5$ & $p<0.001$ & & $p=0.009$ & $p=0.043$ & \\
\hline $3 v 6$ & $p<0.001$ & & $p<0.001$ & $p<0.001$ & $p<0.001$ \\
\hline $4 v 5$ & & & $p<0.001$ & $p<0.001$ & $p<0.001$ \\
\hline $4 v 6$ & & & $p<0.001$ & $p<0.001$ & $p=0.021$ \\
\hline $5 v 6$ & & & $p<0.001$ & $p<0.001$ & $p<0.001$ \\
\hline
\end{tabular}


Table 24. Task 06-06 Bldg HPB02 by Zone

\begin{tabular}{|c|c|c|c|c|c|}
\hline \multirow{2}{*}{ Zone Comparison } & \multicolumn{5}{|c|}{ Variable } \\
\hline & Temp & RelHumidity & DraftRate0_1m & DraftRate0_6m & DraftRate1_1m \\
\hline Main Effect & $\begin{array}{c}F=650.932 \\
p<0.001\end{array}$ & $\begin{array}{c}F=388.828 \\
p<0.001\end{array}$ & $\begin{array}{c}F=28.460 \\
p<0.001\end{array}$ & $\begin{array}{c}F=75.318 \\
p<0.001\end{array}$ & $\begin{array}{c}F=175.963 \\
p<0.001\end{array}$ \\
\hline 0v1 & excluded & excluded & excluded & excluded & excluded \\
\hline $0 \mathrm{v} 2$ & $p<0.001$ & $p<0.001$ & excluded & excluded & excluded \\
\hline 0v3 & $p<0.001$ & $p<0.001$ & excluded & excluded & excluded \\
\hline $0 v 4$ & $p<0.001$ & $p<0.001$ & excluded & excluded & excluded \\
\hline 0v5 & $p<0.001$ & $p<0.001$ & excluded & excluded & excluded \\
\hline 0v6 & $p<0.001$ & $p<0.001$ & excluded & excluded & excluded \\
\hline $1 \mathrm{v} 2$ & excluded & excluded & excluded & excluded & excluded \\
\hline 1v3 & excluded & excluded & excluded & excluded & excluded \\
\hline $1 v 4$ & excluded & excluded & excluded & excluded & excluded \\
\hline $1 v 5$ & excluded & excluded & excluded & excluded & excluded \\
\hline $1 v 6$ & excluded & excluded & excluded & excluded & excluded \\
\hline $2 v 3$ & & $p<0.001$ & & $p<0.001$ & $p<0.001$ \\
\hline $2 v 4$ & & & & $p<0.001$ & $p<0.001$ \\
\hline $2 v 5$ & & & $p<0.001$ & & $p=0.027$ \\
\hline $2 v 6$ & $p<0.001$ & & $p<0.001$ & & $p<0.001$ \\
\hline $3 v 4$ & $p=0.002$ & $p<0.001$ & & $p=0.001$ & $p<0.001$ \\
\hline $3 v 5$ & & $p<0.001$ & $p<0.001$ & $p<0.001$ & $p<0.001$ \\
\hline $3 v 6$ & $p<0.001$ & $p<0.001$ & & $p<0.001$ & $p<0.001$ \\
\hline $4 v 5$ & $p=0.001$ & & $p<0.001$ & $p<0.001$ & $p<0.001$ \\
\hline $4 v 6$ & $p<0.001$ & & $p=0.013$ & $p<0.001$ & $p<0.001$ \\
\hline $5 v 6$ & $p=0.001$ & & $p<0.001$ & $p<0.001$ & $p<0.001$ \\
\hline
\end{tabular}


Table 25. Task 06-06 Bldg HPB03 by Zone

\begin{tabular}{|c|c|c|c|c|c|}
\hline \multirow{2}{*}{ Zone Comparison } & \multicolumn{5}{|c|}{ Variable } \\
\hline & Temp & RelHumidity & DraftRate0_1m & DraftRate0_6m & DraftRate1_1m \\
\hline Main Effect & $\begin{array}{c}F=68.317 \\
p<0.001\end{array}$ & $\begin{array}{c}F=38.522 \\
p<0.001\end{array}$ & $\begin{array}{c}F=114.101 \\
p<0.001\end{array}$ & $\begin{array}{c}F=54.885 \\
p<0.001\end{array}$ & $\begin{array}{c}F=36.215 \\
p<0.001\end{array}$ \\
\hline 0v1 & $p<0.001$ & $p<0.001$ & excluded & excluded & excluded \\
\hline 0v2 & $p<0.001$ & $p<0.001$ & excluded & excluded & excluded \\
\hline 0v3 & $p<0.001$ & $p<0.001$ & excluded & excluded & excluded \\
\hline 0v4 & $p<0.001$ & $p<0.001$ & excluded & excluded & excluded \\
\hline 0v5 & $p<0.001$ & $p<0.001$ & excluded & excluded & excluded \\
\hline 0v6 & $p<0.001$ & $p<0.001$ & excluded & excluded & excluded \\
\hline 1v2 & $p<0.001$ & & & & \\
\hline 1v3 & $p<0.001$ & & $p<0.001$ & $p<0.001$ & $p<0.001$ \\
\hline $1 v 4$ & $p<0.001$ & & $p<0.001$ & & $p=0.027$ \\
\hline 1v5 & $p<0.001$ & & $p=0.001$ & & \\
\hline $1 v 6$ & $p<0.001$ & & $p<0.001$ & $p<0.001$ & $p<0.001$ \\
\hline $2 v 3$ & & & $p<0.001$ & $p<0.001$ & $p<0.001$ \\
\hline $2 v 4$ & $p<0.001$ & & $p<0.001$ & & $p<0.001$ \\
\hline $2 v 5$ & & & $p<0.001$ & & \\
\hline $2 v 6$ & $p=0.008$ & & $p<0.001$ & $p<0.001$ & $p<0.001$ \\
\hline $3 v 4$ & & & $p<0.001$ & $p<0.001$ & $p<0.001$ \\
\hline $3 v 5$ & $p=0.002$ & & & $p<0.001$ & $p<0.001$ \\
\hline $3 v 6$ & & & $p<0.001$ & & $p<0.001$ \\
\hline $4 v 5$ & $p<0.001$ & & $p<0.001$ & & \\
\hline $4 v 6$ & & & $p<0.001$ & $p<0.001$ & \\
\hline $5 v 6$ & $p<0.001$ & & $p<0.001$ & $p<0.001$ & $p=0.013$ \\
\hline st-Hoc via & T3 & & & & \\
\hline
\end{tabular}


Table 26. Task 06-06 Bldg HPB04 by Zone

\begin{tabular}{|c|c|c|c|c|c|}
\hline \multirow{2}{*}{ Zone Comparison } & \multicolumn{5}{|c|}{ Variable } \\
\hline & Temp & RelHumidity & DraftRate0_1m & DraftRate0_6m & DraftRate1_1m \\
\hline Main Effect & $\begin{array}{c}F=3.788 \\
p=0.001\end{array}$ & $\begin{array}{c}F=71.615 \\
p<0.001\end{array}$ & $\begin{array}{c}F=35.802 \\
p<0.001\end{array}$ & $\begin{array}{c}F=100.384 \\
p<0.001\end{array}$ & $\begin{array}{c}F=87.252 \\
p<0.001\end{array}$ \\
\hline 0v1 & & $p<0.001$ & excluded & excluded & excluded \\
\hline 0v2 & & $p<0.001$ & excluded & excluded & excluded \\
\hline 0v3 & & $p<0.001$ & excluded & excluded & excluded \\
\hline 0v4 & & $p<0.001$ & excluded & excluded & excluded \\
\hline 0v5 & & $p<0.001$ & excluded & excluded & excluded \\
\hline $0 v 6$ & & $p<0.001$ & excluded & excluded & excluded \\
\hline $1 \mathrm{v} 2$ & $p=0.047$ & $p<0.001$ & $p=0.020$ & & $p<0.001$ \\
\hline $1 v 3$ & & $p<0.001$ & & $p=0.001$ & \\
\hline $1 v 4$ & $p<0.001$ & $p<0.001$ & $p<0.001$ & $p<0.001$ & $p=0.003$ \\
\hline $1 v 5$ & $p=0.002$ & $p<0.001$ & & $p<0.001$ & $p<0.001$ \\
\hline $1 v 6$ & $p<0.001$ & $p<0.001$ & & $p<0.001$ & $p=0.008$ \\
\hline $2 v 3$ & & $p<0.001$ & $p=0.001$ & $p<0.001$ & $p<0.001$ \\
\hline $2 v 4$ & $p<0.001$ & $p<0.001$ & $p<0.001$ & $p<0.001$ & $p<0.001$ \\
\hline $2 v 5$ & & $p<0.001$ & $p=0.006$ & $p<0.001$ & $p<0.001$ \\
\hline $2 v 6$ & $p<0.001$ & $p<0.001$ & $p<0.001$ & $p<0.001$ & $p<0.001$ \\
\hline $3 v 4$ & $p<0.001$ & $p<0.001$ & $p<0.001$ & $p<0.001$ & $p=0.006$ \\
\hline $3 v 5$ & $p=0.004$ & $p<0.001$ & & $p<0.001$ & $p<0.001$ \\
\hline $3 v 6$ & $p<0.001$ & $p<0.001$ & & $p=0.005$ & $p=0.010$ \\
\hline $4 v 5$ & & & $p<0.001$ & & $p<0.001$ \\
\hline $4 v 6$ & $p<0.001$ & $p<0.001$ & $p<0.001$ & $p=0.001$ & \\
\hline $5 v 6$ & $p<0.001$ & $p<0.001$ & & & $p<0.001$ \\
\hline${ }^{*}$ All Post-Hoc via D & T3 & & & & \\
\hline
\end{tabular}


Table 27. Task 06-06 Bldg HPB05 by Zone

\begin{tabular}{|c|c|c|c|c|c|}
\hline \multirow{2}{*}{ Zone Comparison } & \multicolumn{5}{|c|}{ Variable } \\
\hline & Temp & RelHumidity & DraftRate0_1m & DraftRate0_6m & DraftRate1_1m \\
\hline Main Effect & $\begin{array}{c}F=98.913 \\
p<0.001\end{array}$ & n.s. & $\begin{array}{c}F=8.089 \\
p<0.001\end{array}$ & $\begin{array}{c}F=12.438 \\
p<0.001\end{array}$ & $\begin{array}{c}F=5.429 \\
p<0.001\end{array}$ \\
\hline 0v1 & $p<0.001$ & & excluded & excluded & excluded \\
\hline 0v2 & $p<0.001$ & & excluded & excluded & excluded \\
\hline 0v3 & $p<0.001$ & & excluded & excluded & excluded \\
\hline 0v4 & $p<0.001$ & & excluded & excluded & excluded \\
\hline 0v5 & excluded & & excluded & excluded & excluded \\
\hline $0 v 6$ & $p<0.001$ & & excluded & excluded & excluded \\
\hline $1 v 2$ & $p<0.001$ & & $p=0.049$ & $p<0.001$ & \\
\hline $1 v 3$ & $p=0.007$ & & & & \\
\hline 1v4 & $p=0.022$ & & & & \\
\hline $1 v 5$ & excluded & & $p=0.002$ & $p<0.001$ & \\
\hline $1 v 6$ & $p<0.001$ & & & $p<0.001$ & \\
\hline $2 v 3$ & $p<0.001$ & & & $p<0.001$ & $p<0.001$ \\
\hline $2 v 4$ & $p<0.001$ & & $p=0.023$ & $p=0.011$ & \\
\hline $2 v 5$ & excluded & & & & \\
\hline $2 v 6$ & $p<0.001$ & & $p=0.008$ & & \\
\hline $3 v 4$ & & & & & $p=0.005$ \\
\hline $3 v 5$ & excluded & & $p=0.005$ & $p=0.002$ & \\
\hline $3 v 6$ & $p<0.001$ & & & $p<0.001$ & $p<0.001$ \\
\hline $4 v 5$ & excluded & & $p=0.001$ & & \\
\hline $4 v 6$ & $p=0.025$ & & & & \\
\hline $5 v 6$ & excluded & & $p<0.001$ & & \\
\hline \multicolumn{6}{|c|}{ *All Post-Hoc via Dunnett's T3 } \\
\hline
\end{tabular}


Table 28. Task 06-06 Bldg HPB06 by Zone

\begin{tabular}{|c|c|c|c|c|c|}
\hline \multirow{2}{*}{ Zone Comparison } & \multicolumn{5}{|c|}{ Variable } \\
\hline & Temp & RelHumidity & DraftRate0_1m & DraftRate0_6m & DraftRate1_1m \\
\hline Main Effect & $\begin{array}{c}F=393.845 \\
p<0.001\end{array}$ & $\begin{array}{c}F=165.078 \\
p<0.001\end{array}$ & $\begin{array}{c}F=96.366 \\
p<0.001\end{array}$ & $\begin{array}{c}F=56.382 \\
p<0.001\end{array}$ & $\begin{array}{c}F=68.829 \\
p<0.001\end{array}$ \\
\hline 0v1 & $p<0.001$ & $p<0.001$ & excluded & excluded & excluded \\
\hline 0v2 & $p<0.001$ & $p<0.001$ & excluded & excluded & excluded \\
\hline 0v3 & $p<0.001$ & $p<0.001$ & excluded & excluded & excluded \\
\hline 0v4 & $p<0.001$ & $p<0.001$ & excluded & excluded & excluded \\
\hline 0v5 & $p<0.001$ & $p<0.001$ & excluded & excluded & excluded \\
\hline $0 v 6$ & $p<0.001$ & $p<0.001$ & excluded & excluded & excluded \\
\hline $1 v 2$ & & $p<0.001$ & & $p<0.001$ & $p<0.001$ \\
\hline $1 v 3$ & $p<0.001$ & $p=0.005$ & $p=0.001$ & & $p<0.001$ \\
\hline $1 v 4$ & $p<0.001$ & $p<0.001$ & $p<0.001$ & $p<0.001$ & \\
\hline $1 v 5$ & $p<0.001$ & $p<0.001$ & $p<0.001$ & $p<0.001$ & $p<0.001$ \\
\hline $1 v 6$ & $p<0.001$ & $p<0.001$ & $p<0.001$ & $p=0.020$ & \\
\hline $2 v 3$ & & & & $p<0.001$ & $p<0.001$ \\
\hline 2v4 & $p=0.014$ & & $p<0.001$ & $p<0.001$ & \\
\hline $2 v 5$ & $p<0.001$ & $p<0.001$ & $p<0.001$ & & $p<0.001$ \\
\hline $2 v 6$ & $p<0.001$ & $p<0.001$ & & $p<0.001$ & $p=0.001$ \\
\hline $3 v 4$ & & & $p<0.001$ & $p<0.001$ & $p=0.001$ \\
\hline $3 v 5$ & $p<0.001$ & $p=0.009$ & $p<0.001$ & $p<0.001$ & $p=0.016$ \\
\hline $3 v 6$ & $p<0.001$ & $p<0.001$ & & & $p<0.001$ \\
\hline $4 v 5$ & $p<0.001$ & & $p<0.001$ & $p=0.001$ & $p<0.001$ \\
\hline $4 v 6$ & $p<0.001$ & $p<0.001$ & $p<0.001$ & & \\
\hline $5 v 6$ & $p<0.001$ & $p<0.001$ & $p<0.001$ & $p<0.001$ & $p<0.001$ \\
\hline *All Post-Hoc via Du & s T3 & & & & \\
\hline
\end{tabular}


Table 29. Task 06-06 Bldg HPB07 by Zone

\begin{tabular}{|c|c|c|c|c|c|}
\hline \multirow{2}{*}{ Zone Comparison } & \multicolumn{5}{|c|}{ Variable } \\
\hline & Temp & RelHumidity & DraftRate0_1m & DraftRate0_6m & DraftRate1_1m \\
\hline Main Effect & $\begin{array}{c}F=314.834 \\
p<0.001\end{array}$ & $\begin{array}{c}F=330.545 \\
p<0.001\end{array}$ & $\begin{array}{c}F=67.067 \\
p<0.001\end{array}$ & $\begin{array}{c}F=100.246 \\
p<0.001\end{array}$ & $\begin{array}{c}F=88.516 \\
p<0.001\end{array}$ \\
\hline 0v1 & $p<0.001$ & $p<0.001$ & excluded & excluded & excluded \\
\hline 0v2 & $p<0.001$ & $p<0.001$ & excluded & excluded & excluded \\
\hline 0v3 & $p<0.001$ & $p<0.001$ & excluded & excluded & excluded \\
\hline 0v4 & $p<0.001$ & $p<0.001$ & excluded & excluded & excluded \\
\hline 0v5 & $p<0.001$ & $p<0.001$ & excluded & excluded & excluded \\
\hline $0 v 6$ & $p<0.001$ & $p<0.001$ & excluded & excluded & excluded \\
\hline $1 v 2$ & & $p<0.001$ & & $p<0.001$ & $p=0.004$ \\
\hline $1 v 3$ & $p<0.001$ & $p<0.001$ & $p<0.001$ & $p<0.001$ & $p<0.001$ \\
\hline 1v4 & $p<0.001$ & $p<0.001$ & $p<0.001$ & & $p<0.001$ \\
\hline $1 v 5$ & $p<0.001$ & $p<0.001$ & $p=0.007$ & $p<0.001$ & $p<0.001$ \\
\hline $1 v 6$ & $p<0.001$ & $p<0.001$ & & & $p<0.001$ \\
\hline $2 v 3$ & & $p<0.001$ & $p<0.001$ & $p<0.001$ & $p<0.001$ \\
\hline $2 v 4$ & $p<0.001$ & $p<0.001$ & $p<0.001$ & $p<0.001$ & $p<0.001$ \\
\hline 2v5 & $p<0.001$ & $p<0.001$ & $p=0.001$ & $p<0.001$ & $p<0.001$ \\
\hline $2 v 6$ & $p<0.001$ & & & $p=0.012$ & $p<0.001$ \\
\hline $3 v 4$ & $p<0.001$ & $p<0.001$ & $p<0.001$ & $p<0.001$ & $p<0.001$ \\
\hline $3 v 5$ & $p<0.001$ & & & & \\
\hline $3 v 6$ & $p=0.041$ & $p<0.001$ & $p<0.001$ & $p=0.004$ & $p<0.001$ \\
\hline $4 v 5$ & $p<0.001$ & $p<0.001$ & $p<0.001$ & $p<0.001$ & $p=0.012$ \\
\hline $4 v 6$ & $p<0.001$ & $p<0.001$ & $p<0.001$ & & \\
\hline $5 v 6$ & $p<0.001$ & $p<0.001$ & $p=0.004$ & $p<0.001$ & \\
\hline *All Post-Hoc via & s T3 & & & & \\
\hline
\end{tabular}


Table 30. Task 06-06 Bldg HPB08 by Zone

\begin{tabular}{|c|c|c|c|c|c|}
\hline \multirow{2}{*}{ Zone Comparison } & \multicolumn{5}{|c|}{ Variable } \\
\hline & Temp & RelHumidity & DraftRate0_1m & DraftRate0_6m & DraftRate1_1m \\
\hline Main Effect & $\begin{array}{c}F=105.356 \\
p<0.001\end{array}$ & $\begin{array}{c}F=14.540 \\
p<0.001\end{array}$ & $\begin{array}{c}F=51.753 \\
p<0.001\end{array}$ & $\begin{array}{c}F=134.952 \\
p<0.001\end{array}$ & $\begin{array}{c}F=121.179 \\
p<0.001\end{array}$ \\
\hline 0v1 & $p<0.001$ & $p=0.040$ & excluded & excluded & excluded \\
\hline 0v2 & $p<0.001$ & $p=0.034$ & excluded & excluded & excluded \\
\hline 0v3 & $p<0.001$ & $p<0.001$ & excluded & excluded & excluded \\
\hline 0v4 & $p<0.001$ & $p=0.001$ & excluded & excluded & excluded \\
\hline 0v5 & $p<0.001$ & & excluded & excluded & excluded \\
\hline $0 v 6$ & $p<0.001$ & & excluded & excluded & excluded \\
\hline $1 v 2$ & & & $p<0.001$ & $p<0.001$ & $p<0.001$ \\
\hline $1 v 3$ & & $p<0.001$ & $p<0.001$ & $p<0.001$ & \\
\hline $1 v 4$ & $p<0.001$ & $p=0.001$ & $p<0.001$ & $p=0.034$ & $p<0.001$ \\
\hline 1v5 & & & $p<0.001$ & & \\
\hline $1 v 6$ & & & & $p<0.001$ & $p<0.001$ \\
\hline $2 v 3$ & & $p<0.001$ & $p=0.027$ & $p<0.001$ & $p=0.006$ \\
\hline $2 v 4$ & $p<0.001$ & $p=0.001$ & & $p<0.001$ & $p<0.001$ \\
\hline 2v5 & & & $p<0.001$ & $p<0.001$ & $p<0.001$ \\
\hline $2 v 6$ & & & $p<0.001$ & $p<0.001$ & $p<0.001$ \\
\hline $3 v 4$ & $p<0.001$ & & & $p<0.001$ & $p<0.001$ \\
\hline $3 v 5$ & & $p<0.001$ & & $p<0.001$ & $p=0.035$ \\
\hline $3 v 6$ & & $p<0.001$ & $p<0.001$ & $p<0.001$ & $p<0.001$ \\
\hline $4 v 5$ & $p<0.001$ & $p<0.001$ & $p=0.033$ & $p<0.001$ & $p<0.001$ \\
\hline $4 v 6$ & $p<0.001$ & $p<0.001$ & $p<0.001$ & $p<0.001$ & $p=0.023$ \\
\hline $5 v 6$ & & & $p<0.001$ & $p<0.001$ & $p<0.001$ \\
\hline & s T3 & & & & \\
\hline
\end{tabular}


Table 31. Task 06-06 Bldg HPB09 by Zone

\begin{tabular}{|c|c|c|c|c|c|}
\hline \multirow{2}{*}{ Zone Comparison } & \multicolumn{5}{|c|}{ Variable } \\
\hline & Temp & RelHumidity & DraftRate0_1m & DraftRate0_6m & DraftRate1_1m \\
\hline Main Effect & $\begin{array}{c}F=166.314 ; \\
p<0.001\end{array}$ & $\begin{array}{c}F=125.504 \\
p<0.001\end{array}$ & $\begin{array}{c}F=242.549 \\
p<0.001\end{array}$ & $\begin{array}{c}F=431.348 \\
p<0.001\end{array}$ & $\begin{array}{c}F=338.979 \\
p<0.001\end{array}$ \\
\hline 0v1 & $p<0.001$ & $p<0.001$ & excluded & excluded & excluded \\
\hline 0v2 & $p<0.001$ & $p<0.001$ & excluded & excluded & excluded \\
\hline 0v3 & $p<0.001$ & $p<0.001$ & excluded & excluded & excluded \\
\hline 0v4 & $p<0.001$ & $p<0.001$ & excluded & excluded & excluded \\
\hline 0v5 & $p<0.001$ & $p<0.001$ & excluded & excluded & excluded \\
\hline $0 v 6$ & $p<0.001$ & $p<0.001$ & excluded & excluded & excluded \\
\hline $1 v 2$ & $p<0.001$ & $p<0.001$ & $p<0.001$ & $p<0.001$ & $p<0.001$ \\
\hline $1 v 3$ & & $p=0.042$ & $p<0.001$ & & $p<0.001$ \\
\hline $1 v 4$ & $p<0.001$ & & $p<0.001$ & $p<0.001$ & $p<0.001$ \\
\hline $1 v 5$ & $p<0.001$ & & $p=0.001$ & $p<0.001$ & $p<0.001$ \\
\hline $1 v 6$ & & & $p<0.001$ & $p<0.001$ & $p<0.001$ \\
\hline $2 v 3$ & $p<0.001$ & $p=0.010$ & $p<0.001$ & $p<0.001$ & $p<0.001$ \\
\hline $2 v 4$ & $p<0.001$ & $p<0.001$ & $p<0.001$ & & $p<0.001$ \\
\hline 2v5 & $p<0.001$ & $p<0.001$ & $p<0.001$ & $p<0.001$ & \\
\hline $2 v 6$ & $p<0.001$ & $p<0.001$ & $p<0.001$ & $p<0.001$ & $p<0.001$ \\
\hline $3 v 4$ & $p<0.001$ & & $p<0.001$ & $p<0.001$ & $p<0.001$ \\
\hline $3 v 5$ & $p<0.001$ & & $p=0.008$ & $p<0.001$ & $p<0.001$ \\
\hline $3 v 6$ & $p=0.001$ & $p<0.001$ & $p<0.001$ & $p<0.001$ & $p<0.001$ \\
\hline $4 v 5$ & & & $p<0.001$ & $p<0.001$ & $p<0.001$ \\
\hline $4 v 6$ & $p<0.001$ & $p=0.038$ & $p<0.001$ & $p<0.001$ & $p<0.001$ \\
\hline $5 v 6$ & $p<0.001$ & & $p<0.001$ & & $p<0.001$ \\
\hline
\end{tabular}


Table 32. Task 06-06 Bldg HPB10 by Zone

\begin{tabular}{|c|c|c|c|c|c|}
\hline \multirow{2}{*}{ Zone Comparison } & \multicolumn{5}{|c|}{ Variable } \\
\hline & Temp & RelHumidity & DraftRate0_1m & DraftRate0_6m & DraftRate1_1m \\
\hline Main Effect & $\begin{array}{c}F=33.683 \\
p<0.001\end{array}$ & $\begin{array}{c}F=15.261 \\
p<0.001\end{array}$ & $\begin{array}{c}F=76.078 \\
p<0.001\end{array}$ & $\begin{array}{c}F=128.145 \\
p<0.001\end{array}$ & $\begin{array}{c}F=83.478 \\
p<0.001\end{array}$ \\
\hline 0v1 & & & excluded & excluded & excluded \\
\hline 0v2 & & & excluded & excluded & excluded \\
\hline 0v3 & $p=0.009$ & & excluded & excluded & excluded \\
\hline 0v4 & $p=0.003$ & & excluded & excluded & excluded \\
\hline 0v5 & $p<0.001$ & & excluded & excluded & excluded \\
\hline $0 v 6$ & & & excluded & excluded & excluded \\
\hline $1 v 2$ & $p<0.001$ & $p<0.001$ & $p<0.001$ & $p<0.001$ & $p<0.001$ \\
\hline $1 v 3$ & $p=0.025$ & $p<0.001$ & $p<0.001$ & $p<0.001$ & $p<0.001$ \\
\hline $1 v 4$ & $p<0.001$ & $p<0.001$ & $p<0.001$ & $p<0.001$ & $p=0.030$ \\
\hline $1 v 5$ & $p<0.001$ & $p<0.001$ & & & \\
\hline $1 v 6$ & & & $p<0.001$ & $p<0.001$ & \\
\hline $2 v 3$ & $p<0.001$ & $p<0.001$ & $p<0.001$ & & \\
\hline $2 v 4$ & $p<0.001$ & $p<0.001$ & & $p<0.001$ & $p<0.001$ \\
\hline $2 v 5$ & $p<0.001$ & $p<0.001$ & $p<0.001$ & $p<0.001$ & $p<0.001$ \\
\hline $2 v 6$ & $p<0.001$ & $p<0.001$ & $p<0.001$ & & $p<0.001$ \\
\hline $3 v 4$ & & $p<0.001$ & $p<0.001$ & $p<0.001$ & $p<0.001$ \\
\hline $3 v 5$ & $p<0.001$ & & $p<0.001$ & $p<0.001$ & $p<0.001$ \\
\hline $3 v 6$ & & $p=0.002$ & & & $p<0.001$ \\
\hline $4 v 5$ & $p<0.001$ & $p<0.001$ & $p<0.001$ & $p<0.001$ & \\
\hline $4 v 6$ & $p=0.002$ & & $p=0.003$ & $p<0.001$ & \\
\hline $5 v 6$ & $p<0.001$ & $p=0.001$ & $p<0.001$ & $p<0.001$ & \\
\hline \multicolumn{6}{|c|}{ *All Post-Hoc via Dunnett's T3 } \\
\hline
\end{tabular}




\subsection{AIRBORNE AND SURFACE-ASSOCIATED MOLD}

For more salient interpretation, mold variables were $\log 10$-transformed prior to examination. Statistical tests were conducted for each of the three different measures: airborne culturable fungi, microscopically examined non-culturable airborne fungal spores, and vacuum-collected surface dust. Univariate analysis of variance (ANOVA) was used to determine the presence or absence of a main effect among the three days or the six indoor zones. If a main effect was observed, post-hoc analysis was performed to determine which specific days or zones differed through either Bonferroni's test or Dunnett's T3 test for assumption of equal variance or unequal variance, respectively.

The statistical findings are summarized in the following tables. No statistical differences $(\mathrm{p} \geq 0.05)$ were found by day for airborne culturable fungi or for airborne non-culturable spores. However, some differences $(p<0.05)$ were found by day (for Tuesday vs. Wednesday; $p=0.013$ ) for the surface-associated fungi (Table 33).

Table 33. . Statistical Results by Day for All Tasks for Airborne Culturable Fungi, Airborne Non-culturable Spores, and Surfaceassociated Fungi

\begin{tabular}{|c|c|c|c|}
\hline \multirow{2}{*}{ Comparison } & \multicolumn{3}{|c|}{ Mold Parameter } \\
\cline { 2 - 4 } & Airborne Culturable Fungi & Airborne Non-Culturable Spores & Surface-associated Fungi \\
\hline Main Effect & $(\mathrm{df}=\mathbf{2 , 1 9 6 4 )}$ & $(\mathbf{d f}=\mathbf{2 , 2 5 5 8 )}$ & $\mathbf{( d f = 2 , 3 1 5 4 )}$ \\
\hline Tue vs. Wed & n.s. & n.s. & $\mathrm{F}=\mathbf{4 . 1 2 , p = . 0 1 6}$ \\
\hline Wed vs. Thu & & & $\mathrm{P}=\mathbf{0 . 0 1 3}$ \\
\hline Tue vs. Thu & & & n.s. \\
\hline *Post-Hoc via Bonferroni & & & n.s. \\
\hline
\end{tabular}


Selection of indoor zones (1-6) was independently selected within each task; zones were not related by location across tasks. Therefore, no comparison would be valid across zones for the 30 buildings and statistical analysis by zone was only conducted within each task. No statistically significant differences $(\mathrm{p} \geq 0.05)$ were found by-zone for Task 1 airborne culturable data. Statistically significant differences $(p<0.05)$ were found between outdoor locations (zone 0$)$ and indoor zones 1-6 for Task 1 airborne nonculturable spores (Table 34), but these differences $(\mathrm{p}=0.043)$ were only noted for one indoor location comparison (zone 1 vs. 4) for Task 1 airborne non-culturable fungi.

Table 34. Task 1 Office Buildings by Zone Statistical Results for Airborne Non-culturable Spores

\begin{tabular}{|c|c|c|c|c|c|c|c|c|c|c|}
\hline \multirow{2}{*}{$\begin{array}{c}\text { Zone } \\
\text { Comparison }\end{array}$} & \multicolumn{10}{|c|}{ Building ID } \\
\hline & 001 & 002 & 003 & 004 & 005 & 006 & 007 & 008 & 009 & 010 \\
\hline Main Effect & n.s. & $\begin{array}{l}F=5.801 \\
p=0.000\end{array}$ & $\begin{array}{l}F=4.970 \\
p=0.000\end{array}$ & $\begin{array}{l}F=3.047 \\
p=0.012\end{array}$ & $\begin{array}{l}F=5.273 \\
p=0.000\end{array}$ & $\begin{array}{l}F=8.429 \\
p=0.000\end{array}$ & $\begin{array}{c}F=12.569 \\
p=0.000\end{array}$ & $\begin{array}{c}F=12.678 \\
p=0.000\end{array}$ & $\begin{array}{l}F=3.873 \\
p=0.004\end{array}$ & n.s. \\
\hline 0v1 & & & & & & 0.000 & 0.000 & 0.000 & & \\
\hline 0v2 & & 0.000 & 0.000 & & 0.000 & 0.000 & 0.000 & 0.001 & 0.000 & \\
\hline 0v3 & & 0.010 & 0.000 & & 0.002 & 0.000 & 0.000 & 0.000 & 0.000 & \\
\hline 0v4 & & 0.000 & 0.000 & & 0.031 & 0.000 & 0.000 & 0.000 & 0.003 & \\
\hline 0v5 & & 0.000 & 0.003 & & 0.003 & 0.000 & 0.000 & 0.000 & 0.000 & \\
\hline 0v6 & & 0.002 & 0.000 & & 0.000 & 0.000 & 0.000 & 0.000 & 0.000 & \\
\hline $1 \mathrm{v} 2$ & & & & & & & & & & \\
\hline $1 v 3$ & & & & & & & & & & \\
\hline $1 v 4$ & & & & & & 0.043 & & & & \\
\hline $1 v 5$ & & & & & & & & & & \\
\hline $1 v 6$ & & & & & & & & & & \\
\hline $2 v 3$ & & & & & & & & & & \\
\hline $2 v 4$ & & & & & & & & & & \\
\hline $2 v 5$ & & & & & & & & & & \\
\hline $2 v 6$ & & & & & & & & & & \\
\hline $3 v 4$ & & & & & & & & & & \\
\hline $3 v 5$ & & & & & & & & & & \\
\hline $3 v 6$ & & & & & & & & & & \\
\hline $4 v 5$ & & & & & & & & & & \\
\hline $4 \mathrm{v} 6$ & & & & & & & & & & \\
\hline $5 v 6$ & & & & & & & & & & \\
\hline
\end{tabular}




\section{STATISTICAL ANALYSIS AND RESULTS}

Statistically significant differences $(\mathrm{p}<0.05)$ were found during analysis by indoor zone for the Task 1 surface-associated fungal data for 6 of the 10 buildings (Table 35).

Table 35. Task 1 Office Buildings by Zone Statistical Results for Surface-associated Fungi.

\begin{tabular}{|c|c|c|c|c|c|c|c|c|c|c|}
\hline \multirow{2}{*}{$\begin{array}{c}\text { Zone } \\
\text { Comparison }\end{array}$} & \multicolumn{10}{|c|}{ Building } \\
\hline & 001 & 002 & 003 & 004 & 005 & 006 & 007 & 008 & 009 & 010 \\
\hline Main Effect & $\begin{array}{l}F=2.321 \\
p=0.048\end{array}$ & n.s. & n.s. & $\begin{array}{c}F=13.266 \\
p=0.000\end{array}$ & $\begin{array}{l}F=5.107 \\
p=0.000\end{array}$ & $\begin{array}{l}F=7.463 \\
p=0.000\end{array}$ & $\begin{array}{l}F=2.618 \\
p=0.028\end{array}$ & n.s. & $\begin{array}{c}F=11.521 \\
p=0.000\end{array}$ & n.s. \\
\hline 1v2 & & & & & 0.001 & & 0.023 & & & \\
\hline 1v3 & & & & 0.001 & 0.019 & & & & & \\
\hline \multicolumn{11}{|l|}{$1 v 4$} \\
\hline $1 v 5$ & & & & 0.020 & 0.002 & & & & & \\
\hline $1 \mathrm{v} 6$ & & & & & & 0.001 & & & & \\
\hline $2 v 3$ & & & & 0.001 & & & & & 0.000 & \\
\hline $2 v 4$ & & & & & & & & & 0.000 & \\
\hline $2 v 5$ & & & & 0.009 & & & & & 0.000 & \\
\hline $2 v 6$ & & & & & & 0.008 & & & & \\
\hline $3 v 4$ & 0.023 & & & 0.000 & & & & & & \\
\hline \\
\hline $3 v 6$ & & & & 0.000 & & 0.001 & & & 0.000 & \\
\hline $4 v 5$ & & & & 0.000 & & & & & & \\
\hline $4 v 6$ & & & & & & 0.004 & & & 0.000 & \\
\hline $5 v 6$ & & & & 0.007 & & & & & 0.001 & \\
\hline
\end{tabular}


Statistically significant differences $(\mathrm{p}<0.05)$ at indoor zones in airborne culturable fungi were noted for Task 13 data in two of the 10 buildings. The differences were found when comparing zone 1 vs 4 in one school building (Building S5), and in two indoor zone comparisons (zones 2 vs 5 and 2 vs 6) in another school building (Building S2) (Table 36). Differences were also found when comparing ourdoor (zone 0) and indoor zones in several of the buildings.

Table 36. Task 13 Schools by Zone Results for Airborne Culturable Fungi

\begin{tabular}{|c|c|c|c|c|c|c|c|c|c|c|}
\hline \multirow{2}{*}{$\begin{array}{c}\text { Zone } \\
\text { Comparison }\end{array}$} & \multicolumn{10}{|c|}{ Building } \\
\hline & S01 & SO2 & SO3 & S04 & S05 & S06 & S07 & S08 & S09 & S10 \\
\hline Main Effect & $\begin{array}{l}F=2.992 \\
p=0.011\end{array}$ & $\begin{array}{c}F=27.494 \\
p=0.000\end{array}$ & n.s. & n.s. & $\begin{array}{l}F=7.021 \\
p=0.000\end{array}$ & n.s. & $\begin{array}{l}F=2.553 \\
p=0.030\end{array}$ & n.s. & $\begin{array}{l}F=2.755 \\
p=0.019\end{array}$ & $\begin{array}{c}\text { (Table 5), } \\
\text { and } \\
\text { surface- } \\
\text { ass } 0 \mathrm{cn} . \mathrm{s} \text {. }\end{array}$ \\
\hline 0v1 & 0.036 & & & & & & & & & \\
\hline \multicolumn{11}{|l|}{ 0v2 } \\
\hline 0v3 & 0.023 & & & & & & & & & \\
\hline 0v4 & & & & & 0.000 & & 0.005 & & & \\
\hline 0v5 & & 0.000 & & & & & & & & \\
\hline 0v6 & & 0.000 & & & & 0.000 & & & & \\
\hline \multicolumn{11}{|l|}{$1 v 2$} \\
\hline \multicolumn{11}{|l|}{$1 v 3$} \\
\hline $1 v 4$ & & & & & 0.006 & & & & & \\
\hline \multicolumn{11}{|l|}{$1 v 5$} \\
\hline \multicolumn{11}{|l|}{$1 v 6$} \\
\hline \multicolumn{11}{|l|}{$2 \mathrm{v} 3$} \\
\hline $2 v 4$ & & & & & MD & & & & & \\
\hline $2 \mathrm{v} 5$ & & 0.002 & & & & & & & & \\
\hline $2 v 6$ & & 0.002 & & & & & & & & \\
\hline \multicolumn{11}{|l|}{$3 v 4$} \\
\hline \multicolumn{11}{|l|}{$3 v 5$} \\
\hline $3 v 6$ & & & & & & MD & & & & \\
\hline $4 v 5$ & & 0.000 & & & & & & & & \\
\hline $4 v 6$ & & 0.000 & & & & & & & & \\
\hline $5 v 6$ & & MD & & & & & & & & \\
\hline
\end{tabular}




\section{STATISTICAL ANALYSIS AND RESULTS}

While differences were found for indoor vs. outdoors, no statistically significant differences ( $\mathrm{p} \geq 0.05)$ were found for airborne non-culturable spores in the Task 13 indoor zones (1-6) (Table 37).

Table 37. Task 13 Schools by Zone Results for Airborne Non-culturable Spores

\begin{tabular}{|c|c|c|c|c|c|c|c|c|c|c|}
\hline \multirow{2}{*}{$\begin{array}{c}\text { Zone } \\
\text { Comparison }\end{array}$} & \multicolumn{10}{|c|}{ Building } \\
\hline & S01 & S02 & S03 & S04 & S05 & S06 & S07 & S08 & S09 & S10 \\
\hline Main Effect & $\begin{array}{c}F=10.556 \\
p=0.000\end{array}$ & $\begin{array}{l}F=5.308 \\
p=0.000\end{array}$ & $\begin{array}{l}F=3.956 \\
p=0.002\end{array}$ & $\begin{array}{l}F=2.754 \\
p=0.014\end{array}$ & $\begin{array}{l}F=5.045 \\
p=0.000\end{array}$ & $\begin{array}{l}F=3.536 \\
p=0.017\end{array}$ & $\begin{array}{l}F=7.391 \\
p=0.000\end{array}$ & $\begin{array}{l}F=3.456 \\
p=0.003\end{array}$ & $\begin{array}{l}F=4.273 \\
p=0.001\end{array}$ & $\begin{array}{l}F=8.289 \\
p=0.000\end{array}$ \\
\hline 0v1 & 0.000 & & & & 0.006 & & & & & \\
\hline 0v2 & 0.000 & 0.022 & 0.000 & & & & 0.000 & 0.003 & & 0.043 \\
\hline 0v3 & & & & 0.043 & 0.005 & & 0.004 & & 0.004 & 0.016 \\
\hline 0v4 & 0.000 & & & & & 0.000 & 0.000 & & & 0.000 \\
\hline 0v5 & 0.000 & & 0.009 & & 0.000 & & 0.000 & & & 0.000 \\
\hline 0v6 & 0.000 & & & & & & 0.000 & & 0.018 & 0.028 \\
\hline 1v2 & & & & & & & & & & \\
\hline 1v3 & & & & & & & & & & \\
\hline $1 v 4$ & & & & & & & & & & \\
\hline $1 v 5$ & & & & & & & & & & \\
\hline $1 v 6$ & & & & & & & & & & \\
\hline $2 v 3$ & & & & & & & & & & \\
\hline $2 v 4$ & & & & & & & & & & \\
\hline $2 v 5$ & & & & & & & & & & \\
\hline $2 v 6$ & & & & & & & & & & \\
\hline $3 v 4$ & & & & & & & & & & \\
\hline $3 v 5$ & & & & & & & & & & \\
\hline $3 v 6$ & & & & & & & & & & \\
\hline $4 \mathrm{v} 5$ & & & & & & MD & & & & \\
\hline $4 \mathrm{v} 6$ & & & & & & & & & & \\
\hline $5 v 6$ & & & & & & & & & & \\
\hline
\end{tabular}


Statistically significant differences $(\mathrm{p}<0.05)$ were found for surface-associated fungi for Task 13 indoors zones in four of the school buildings (Building S01, S02, S07, and S09) (Table 38).

Table 38. Task 13 Schools by Zone Findings for Surface-associated Fungi

\begin{tabular}{|c|c|c|c|c|c|c|c|c|c|c|}
\hline \multirow{2}{*}{$\begin{array}{c}\text { Zone } \\
\text { Comparison }\end{array}$} & \multicolumn{10}{|c|}{ Building } \\
\hline & S01 & S02 & S03 & S04 & S05 & S06 & S07 & S08 & S09 & S10 \\
\hline Main Effect & $\begin{array}{l}F=4.077 \\
p=0.002\end{array}$ & $\begin{array}{l}F=9.080 \\
p=0.000\end{array}$ & n.s. & n.s. & n.s. & n.s. & $\begin{array}{l}F=3.519 \\
p=0.005\end{array}$ & n.s. & $\begin{array}{l}F=2.786 \\
p=0.020\end{array}$ & n.s. \\
\hline 1v2 & & & & & & & & & & \\
\hline $1 v 3$ & & & & & & & 0.015 & & & \\
\hline 1v4 & & & & & & & & & & \\
\hline 1v5 & & & & & & & & & & \\
\hline $1 v 6$ & & 0.002 & & & & & 0.018 & & & \\
\hline $2 \mathrm{v} 3$ & 0.022 & & & & & & & & & \\
\hline $2 \mathrm{v} 4$ & & & & & & & & & & \\
\hline 2v5 & 0.001 & & & & & & & & & \\
\hline $2 \mathrm{v} 6$ & & 0.000 & & & & & & & 0.012 & \\
\hline $3 v 4$ & & & & & & & & & & \\
\hline $3 v 5$ & & & & & & & & & & \\
\hline $3 v 6$ & & & & & & & & & & \\
\hline $4 \mathrm{v} 5$ & & 0.001 & & & & & & & & \\
\hline $4 \mathrm{v} 6$ & & & & & & & & & & \\
\hline $5 v 6$ & & 0.000 & & & & & & & & \\
\hline
\end{tabular}




\section{STATISTICAL ANALYSIS AND RESULTS}

Although differences were found when comparing outdoor (zone 0) airborne culturable fungi to that found in indoor zones (1-6), no statistically significant differences $(\mathrm{p} \geq 0.05)$ were found by indoor zone (1-6) (Table 39) or for airborne non-culturable spores (Table 40) in the high performance buildings of Task 0606.

Table 39. Task 0606 High Performance Buildings by Zone Results for Airborne Culturable Fungi

\begin{tabular}{|c|c|c|c|c|c|c|c|c|c|c|}
\hline \multirow{2}{*}{$\begin{array}{c}\text { Zone } \\
\text { Comparison }\end{array}$} & \multicolumn{10}{|c|}{ Building } \\
\hline & HPB01 & HPB02 & HPB03 & HPB04 & HРB05 & HPB06 & HPB07 & HPB08 & HPB09 & HPB010 \\
\hline Main Effect & $\begin{array}{l}F=4.115 \\
p=0.001\end{array}$ & $\begin{array}{c}F=20.151 \\
p=0.000\end{array}$ & $\begin{array}{l}F=4.979 \\
p=0.001\end{array}$ & $\begin{array}{l}F=7.264 \\
p=0.000\end{array}$ & n.s. & n.s. & $\begin{array}{l}F=5.954 \\
p=0.000\end{array}$ & $\begin{array}{l}F=3.198 \\
p=0.007\end{array}$ & n.s. & n.s. \\
\hline 0v1 & 0.050 & 0.000 & & 0.001 & & & & & & \\
\hline 0v2 & 0.048 & & 0.002 & 0.000 & & & & & & \\
\hline 0v3 & 0.035 & & & & & & & & & \\
\hline 0v4 & 0.033 & & & 0.000 & & & 0.050 & & & \\
\hline 0v5 & 0.041 & & & 0.000 & & & 0.002 & 0.039 & & \\
\hline \multicolumn{11}{|l|}{$0 v 6$} \\
\hline \multicolumn{11}{|l|}{$1 v 2$} \\
\hline \multicolumn{11}{|l|}{$1 v 3$} \\
\hline \multicolumn{11}{|l|}{$1 \mathrm{v} 4$} \\
\hline \multicolumn{11}{|l|}{$1 v 5$} \\
\hline \multicolumn{11}{|l|}{$1 v 6$} \\
\hline \multicolumn{11}{|l|}{$2 v 3$} \\
\hline \multicolumn{11}{|l|}{$2 v 4$} \\
\hline \multicolumn{11}{|l|}{$2 v 5$} \\
\hline \multicolumn{11}{|l|}{$2 v 6$} \\
\hline \multicolumn{11}{|l|}{$3 v 4$} \\
\hline \multicolumn{11}{|l|}{$3 v 5$} \\
\hline \multicolumn{11}{|l|}{$3 v 6$} \\
\hline \multicolumn{11}{|l|}{$4 v 5$} \\
\hline \multicolumn{11}{|l|}{$4 v 6$} \\
\hline $5 v 6$ & & & & & & & & & & \\
\hline
\end{tabular}


Table 40. Task 0606 High Performance Buildings by Zone Results for Airborne Non-culturable Spores

\begin{tabular}{|c|c|c|c|c|c|c|c|c|c|c|}
\hline \multirow{2}{*}{$\begin{array}{c}\text { Zone } \\
\text { Comparison }\end{array}$} & \multicolumn{10}{|c|}{ Building } \\
\hline & HPB01 & HРB02 & НРВ03 & HРB04 & HРB05 & НРB06 & HPB07 & НРВ08 & HPB09 & НРB010 \\
\hline Main Effect & $\begin{array}{l}F=4.917 \\
p=0.000\end{array}$ & $\begin{array}{l}F=7.565 \\
p=0.000\end{array}$ & $\begin{array}{l}F=7.121 \\
p=0.000\end{array}$ & $\begin{array}{l}F=9.478 \\
p=0.000\end{array}$ & $\begin{array}{c}F=11.081 \\
p=0.000\end{array}$ & $\begin{array}{l}F=5.272 \\
p=0.000\end{array}$ & $\begin{array}{c}F=13.190 \\
p=0.000\end{array}$ & $\begin{array}{c}F=11.441 \\
p=0.000\end{array}$ & $\begin{array}{l}F=6.635 \\
p=0.000\end{array}$ & $\begin{array}{c}F=20.841 \\
p=0.000\end{array}$ \\
\hline 0v1 & 0.005 & & & 0.000 & 0.000 & 0.002 & & 0.018 & & 0.000 \\
\hline 0v2 & 0.000 & & & 0.000 & & 0.015 & & 0.000 & & 0.000 \\
\hline 0v3 & & & & & 0.001 & 0.019 & 0.000 & 0.001 & & 0.000 \\
\hline 0v4 & & 0.000 & & 0.000 & 0.000 & 0.004 & 0.000 & 0.000 & & 0.000 \\
\hline 0v5 & 0.001 & & & 0.000 & 0.000 & & 0.000 & 0.000 & 0.000 & 0.000 \\
\hline $0 v 6$ & 0.000 & & & 0.000 & 0.001 & & 0.000 & 0.001 & & 0.000 \\
\hline 1v2 & & & & & & & & & & \\
\hline $1 \mathrm{v} 3$ & & & & & & & & & & \\
\hline $1 v 4$ & & & & & & & & & & \\
\hline $1 v 5$ & & & & & & & & & & \\
\hline $1 v 6$ & & & & & & & & & & \\
\hline 2v3 & & & & & & & & & & \\
\hline $2 \mathrm{v} 4$ & & & & & & & & & & \\
\hline 2v5 & & & & & & & & & & \\
\hline $2 v 6$ & & & & & & & & & & \\
\hline $3 v 4$ & & & & & & & & & & \\
\hline $3 v 5$ & & & & & & & & & & \\
\hline $3 v 6$ & & & & & & & & & & \\
\hline $4 v 5$ & & & & & & & & & & \\
\hline $4 v 6$ & & & & & & & & & & \\
\hline $5 v 6$ & & & & & & & & & & \\
\hline
\end{tabular}




\section{STATISTICAL ANALYSIS AND RESULTS}

Statistically significant differences $(\mathrm{p}<0.05)$ were found for surface-associated fungi in three of the 10 high performance buildings of Task 0606 (Table 41).

Table 41. Task 0606 High Performance Buildings by Zone Results for Surface-associated Fungi

\begin{tabular}{|c|c|c|c|c|c|c|c|c|c|c|}
\hline \multirow{2}{*}{$\begin{array}{c}\text { Zone } \\
\text { Comparison }\end{array}$} & \multicolumn{10}{|c|}{ Building } \\
\hline & HРB01 & HPB02 & HPB03 & HPB04 & HPB05 & HРB06 & HPB07 & HРB08 & HPB09 & HРB010 \\
\hline Main Effect & $\begin{array}{l}F=4.399 \\
p=0.001\end{array}$ & n.s. & $\begin{array}{l}F=7.605 \\
p=0.000\end{array}$ & n.s. & n.s. & $\begin{array}{l}F=4.105 \\
p=0.002\end{array}$ & n.s. & $\begin{array}{l}F=2.898 \\
p=0.023\end{array}$ & n.s. & n.s. \\
\hline 1v2 & & & & & & & & & & \\
\hline 1v3 & 0.043 & & & & & & & & & \\
\hline $1 \mathrm{v} 4$ & & & & & & & & & & \\
\hline 1v5 & & & & & & & & & & \\
\hline $1 v 6$ & & & & & & 0.023 & & & & \\
\hline $2 \mathrm{v} 3$ & 0.001 & & 0.028 & & & & & & & \\
\hline $2 \mathrm{v} 4$ & & & 0.036 & & & & & & & \\
\hline 2v5 & & & & & & & & & & \\
\hline $2 v 6$ & & & & & & & & & & \\
\hline $3 v 4$ & & & & & & & & & & \\
\hline $3 v 5$ & & & & & & & & & & \\
\hline $3 v 6$ & 0.009 & & 0.008 & & & & & & & \\
\hline $4 \mathrm{v} 5$ & & & & & & & & & & \\
\hline $4 \mathrm{v} 6$ & & & 0.008 & & & & & & & \\
\hline $5 v 6$ & & & & & & & & & & \\
\hline
\end{tabular}

\subsection{LIGHTING}

Multiple measures of each lighting variable within a single day were only performed during Task 1 . Therefore, by-day comparisons were only analyzed for that task. In the case of normally distributed variables, ANOVA was used to determine the presence or absence of a main effect among the three days or the seven zones. These variables were CombChromX, CombCCT, CombCRI, AmbChromY1, and IllumWkSfcDrkst. All remaining variables were found to be non-normally distributed, thus nonparametric Kruskal-Wallis (K-W) tests were performed for: CombChromY, AmbChromX1, AmbCCT1, AmbCRI1, and IllumWkSfcBrtst. If a main effect was observed, post-hoc analysis was performed to determine which specific days or zones differed through either Tukey's Honest Significant Difference (HSD) test or Dunnett's T3 test for assumption of equal variance or unequal variance, respectively.

There were no significant differences $(\mathrm{p} \geq 0.05)$ among the days of collection for all Task 1 office. The statistical results for by-zone comparisons for Task 1 are summarized below, where each table represents either main effect or K-W statistic (Table 42-49). Fields denoted by " +++ " indicate that all zones for a particular building were non-homogenous subsets with equivalent values for each case in all zones, thus 
preventing comparison by ANOVA. These tables show the results from all post-hoc tests if a difference was observed. There were no significant differences by-zone in any variables for Buildings O03, O06, and O10. Therefore, post-hoc analysis was not performed. Fields denoted by "--." indicate that the measures for both zones were equivalent, thus preventing comparison of variance.

By-zone comparisons were analyzed differently for Task 1 as compared to Task 13 and Task 0606. Due to Task 13 and Task 0606 only having a single measurement of each variable for each zone, Chi-square $\left(\chi^{2}\right)$ tests of uniformity were used for variables with values $>1$ and Kolmogorov-Smirnov (K-S) for variables with values <1. By-zone comparisons for Tasks 13 and 0606 are listed in Tables 50-51, respectively. Note that Task 0606 only has analysis for illumination, because all other variables of interest were not collected.

Table 42. Task 1 Office Buildings Lighting by Zone: All Buildings

\begin{tabular}{|c|c|c|c|c|c|}
\hline \multicolumn{6}{|c|}{ ANOVA } \\
\hline \multirow{2}{*}{$\begin{array}{l}\text { Office } \\
\text { Bldg ID }\end{array}$} & \multicolumn{5}{|c|}{ Variable } \\
\hline & CombChromX & CombCCT & CombCRI & AmbChromY1 & IllumWkSfcDrkst \\
\hline 001 & $26.914 ; 0.000$ & $32.597 ; 0.000$ & $49.978 ; 0.000$ & $66.242 ; 0.000$ & $4.708 ; .0013$ \\
\hline 002 & $0.800 ; 0.571$ & $0.882 ; 0.522$ & $0.771 ; 0.588$ & $4.044 ; 0.022$ & $2.501 ; 0.090$ \\
\hline 003 & $0.535 ; 0.746$ & $0.561 ; 0.728$ & $2.533 ; 0.087$ & $0.924 ; 0.498$ & $1.161 ; 0.382$ \\
\hline 004 & $1.816 ; 0.184$ & $1.653 ; 0.220$ & $4.218 ; 0.019$ & $423.755 ; 0.000$ & $0.621 ; 0.687$ \\
\hline 005 & +++ & $3.850 ; 0.026$ & $0.412 ; 0.832$ & +++ & $1.979 ; 0.154$ \\
\hline 006 & $1.544 ; 0.249$ & $1.688 ; 0.212$ & $0.114 ; 0.987$ & +++ & $0.746 ; 0.604$ \\
\hline 007 & $1.037 ; 0.440$ & $0.925 ; 0.498$ & $1.366 ; 0.304$ & +++ & $1.790 ; 0.189$ \\
\hline 008 & $3.941 ; 0.024$ & $19.402 ; 0.000$ & $8.846 ; 0.001$ & +++ & $1.305 ; 0.325$ \\
\hline 009 & $1.718 ; 0.205$ & $1.444 ; 0.278$ & $1.895 ; 0.169$ & +++ & $1.201 ; 0.366$ \\
\hline 010 & $1.937 ; 0.161$ & $1.664 ; 0.217$ & $1.667 ; 0.217$ & +++ & $2.934 ; 0.059$ \\
\hline \multicolumn{6}{|c|}{ Nonparametric Measures } \\
\hline \multirow{2}{*}{$\begin{array}{c}\text { Office } \\
\text { Bldg ID }\end{array}$} & \multicolumn{5}{|c|}{ Variable } \\
\hline & CombChromY & AmbChromX1 & AmbCCT1 & AmbCRI1 & IllumWkSfcBrtst \\
\hline 001 & $13.638 ; 0.018$ & $15.896 ; 0.007$ & $15.489 ; 0.008$ & $14.781 ; 0.011$ & $16.158 ; 0.006$ \\
\hline 002 & $1.234 ; 0.942$ & $11.896 ; 0.036$ & $11.826 ; 0.037$ & $8.149 ; 0.148$ & $3.830 ; 0.574$ \\
\hline 003 & $3.032 ; 0.695$ & $1.112 ; 0.953$ & $2.099 ; 0.835$ & $4.565 ; 0.471$ & $10.006 ; 0.075$ \\
\hline 004 & $7.232 ; 0.204$ & $16.516 ; 0.006$ & $16.516 ; 0.006$ & $17.000 ; 0.004$ & $10.778 ; 0.056$ \\
\hline 005 & $17.000 ; 0.004$ & $17.000 ; 0.004$ & $0.000 ; 1.000$ & $0.000 ; 1.000$ & $9.023 ; 0.108$ \\
\hline 006 & $3.214 ; 0.667$ & $0.000 ; 1.000$ & $0.000 ; 1.000$ & $0.000 ; 1.000$ & $8.953 ; 0.111$ \\
\hline 007 & $4.414 ; 0.491$ & $17.000 ; 0.004$ & $0.000 ; 1.000$ & $0.000 ; 1.000$ & $11.152 ; 0.048$ \\
\hline 008 & $9.047 ; 0.107$ & $0.000 ; 1.000$ & $0.000 ; 1.000$ & $0.000 ; 1.000$ & $13.757 ; 0.017$ \\
\hline 009 & $7.745 ; 0.171$ & $17.000 ; 0.004$ & $17.000 ; 0.004$ & $17.000 ; 0.004$ & $6.591 ; 0.253$ \\
\hline 010 & $9.618 ; 0.087$ & $0.000 ; 1.000$ & $0.000 ; 1.000$ & $0.000 ; 1.000$ & $5.842 ; 0.322$ \\
\hline
\end{tabular}




\section{STATISTICAL ANALYSIS AND RESULTS}

Table 43. Task 1 Office Buildings Lighting by Zone: Building 001

\begin{tabular}{|c|c|c|c|c|c|}
\hline \multicolumn{6}{|c|}{ ANOVA } \\
\hline \multirow{2}{*}{$\begin{array}{c}\text { Zone } \\
\text { Comparison }\end{array}$} & \multicolumn{5}{|c|}{ Variable } \\
\hline & CombChromX & CombCCT & CombCRI & AmbChromY1 & IllumWkSfcDrkst \\
\hline Main Effect & $26.914 ; 0.000$ & $32.597 ; 0.000$ & $49.978 ; 0.000$ & $66.242 ; 0.000$ & $4.708 ; 0.013$ \\
\hline 1 v 2 & & & 0.030 & & \\
\hline 1 v 3 & & & 0.009 & 0.004 & \\
\hline $1 v 4$ & & & -- & & \\
\hline 1 v 5 & & & & 0.022 & \\
\hline $1 \vee 6$ & & & 0.009 & & \\
\hline \multicolumn{6}{|l|}{2 v 3} \\
\hline $2 \vee 4$ & & & & & 0.005 \\
\hline $2 v 5$ & & & & 0.001 & \\
\hline $2 \vee 6$ & & & & & 0.000 \\
\hline $3 \vee 4$ & & & & & 0.007 \\
\hline $3 v 5$ & & & & 0.006 & \\
\hline $3 v 6$ & 0.038 & 0.019 & & & 0.000 \\
\hline $4 \vee 5$ & & & & 0.002 & \\
\hline $4 \vee 6$ & 0.009 & & & & 0.019 \\
\hline $5 \vee 6$ & & & & & 0.037 \\
\hline \multicolumn{6}{|c|}{ Nonparametric Measures } \\
\hline \multirow{2}{*}{$\begin{array}{c}\text { Zone } \\
\text { Comparison }\end{array}$} & \multicolumn{5}{|c|}{ Variable } \\
\hline & CombChromY & AmbChromX1 & AmbCCT1 & AmbCRI1 & IllumWkSfcBrtst \\
\hline K-W Statistic & $13.638 ; 0.018$ & $15.896 ; 0.007$ & $15.489 ; 0.008$ & $14.781 ; 0.011$ & $16.158 ; 0.006$ \\
\hline $1 v 2$ & & & & & 0.015 \\
\hline 1 v 3 & & 0.002 & 0.001 & --- & \\
\hline $1 v 4$ & & & 0.028 & & \\
\hline $1 v 5$ & & 0.002 & 0.001 & & 0.047 \\
\hline 1 v 6 & & & 0.020 & & \\
\hline $2 v 3$ & & & & & \\
\hline $2 \vee 4$ & & & & & \\
\hline $2 v 5$ & & 0.011 & 0.009 & & \\
\hline 2 v 6 & 0.043 & & & & \\
\hline $3 v 4$ & & 0.049 & & & \\
\hline $3 v 5$ & 0.039 & 0.000 & 0.000 & & \\
\hline $3 \vee 6$ & & 0.009 & 0.009 & & \\
\hline 4 v 5 & & 0.000 & 0.000 & 0.024 & \\
\hline 4 v 6 & & 0.000 & & & \\
\hline $5 \vee 6$ & & & 0.000 & & \\
\hline
\end{tabular}


Table 44. Task 1 Office Buildings Lighting by Zone: Building 002

\begin{tabular}{|c|c|c|c|c|c|}
\hline \multicolumn{6}{|c|}{ ANOVA } \\
\hline \multirow[t]{2}{*}{ Zone Comparison } & \multicolumn{5}{|c|}{ Variable } \\
\hline & CombChromX & CombCCT & CombCRI & AmbChromY1 & IllumWkSfcDrkst \\
\hline Main Effect & n.s. & n.s. & n.s. & $4.044 ; 0.022$ & n.s. \\
\hline \multicolumn{6}{|l|}{1 v 2} \\
\hline \multicolumn{6}{|l|}{$1 \vee 3$} \\
\hline \multicolumn{6}{|l|}{$1 \vee 4$} \\
\hline \multicolumn{6}{|l|}{1 v 5} \\
\hline \multicolumn{6}{|l|}{$1 \vee 6$} \\
\hline \multicolumn{6}{|l|}{$2 \vee 3$} \\
\hline \multicolumn{6}{|l|}{$2 \vee 4$} \\
\hline \multicolumn{6}{|l|}{$2 \vee 5$} \\
\hline \multicolumn{6}{|l|}{$2 \vee 6$} \\
\hline \multicolumn{6}{|l|}{$3 \vee 4$} \\
\hline \multicolumn{6}{|l|}{$3 v 5$} \\
\hline $3 v 6$ & & & & 0.041 & \\
\hline \multicolumn{6}{|l|}{4 v 5} \\
\hline \multicolumn{6}{|l|}{$4 \vee 6$} \\
\hline \multicolumn{6}{|l|}{$5 \vee 6$} \\
\hline \multicolumn{6}{|c|}{ Nonparametric Measures } \\
\hline \multirow[t]{2}{*}{ Zone Comparison } & \multicolumn{5}{|c|}{ Variable } \\
\hline & CombChromY & AmbChromX1 & AmbCCT1 & AmbCRI1 & IllumWkSfcBrtst \\
\hline K-W Statistic & n.s. & $11.896 ; 0.036$ & $11.826 ; 0.037$ & n.s. & n.s. \\
\hline \multicolumn{6}{|l|}{1 v 2} \\
\hline \multicolumn{6}{|l|}{$1 \vee 3$} \\
\hline \multicolumn{6}{|l|}{$1 \vee 4$} \\
\hline \multicolumn{6}{|l|}{$1 \vee 5$} \\
\hline \multicolumn{6}{|l|}{$1 \vee 6$} \\
\hline \multicolumn{6}{|l|}{$2 \vee 3$} \\
\hline \multicolumn{6}{|l|}{$2 \vee 4$} \\
\hline $2 \vee 5$ & & & & & \\
\hline $2 v 6$ & & & & & \\
\hline $3 \vee 4$ & & 0.031 & 0.049 & & \\
\hline $3 v 5$ & & & & & \\
\hline $3 v 6$ & & 0.011 & 0.014 & & \\
\hline $4 \vee 5$ & & & & & \\
\hline $4 \vee 6$ & & & & & \\
\hline $5 \vee 6$ & & & & & \\
\hline
\end{tabular}


Table 45. Task 1 Office Buildings Lighting by Zone: Building 004

\begin{tabular}{|c|c|c|c|c|c|}
\hline \multicolumn{6}{|c|}{ ANOVA } \\
\hline \multirow{2}{*}{$\begin{array}{c}\text { Zone } \\
\text { Comparison }\end{array}$} & \multicolumn{5}{|c|}{ Variable } \\
\hline & CombChromX & CombCCT & CombCRI & AmbChromY1 & IllumWkSfcDrkst \\
\hline Main Effect & n.s. & n.s. & $4.218 ; 0.019$ & $423.755 ; 0.000$ & n.s. \\
\hline \multicolumn{6}{|l|}{$1 \vee 2$} \\
\hline 1 v 3 & & & & --- & \\
\hline 1 v 4 & & & & --- & \\
\hline $1 \vee 5$ & & & 0.028 & --- & \\
\hline $1 \vee 6$ & & & & --- & \\
\hline \multicolumn{6}{|l|}{2 v 3} \\
\hline 2 v 4 & & & & 0.040 & \\
\hline $2 \vee 5$ & & & & 0.015 & \\
\hline \multicolumn{6}{|l|}{$2 \vee 6$} \\
\hline 3 v 4 & & & & --- & \\
\hline $3 \vee 5$ & & & & --- & \\
\hline $3 \vee 6$ & & & & --- & \\
\hline $4 v 5$ & & & & --- & \\
\hline $4 \vee 6$ & & & & --- & \\
\hline $5 \vee 6$ & & & & --- & \\
\hline \multicolumn{6}{|c|}{ Nonparametric Measures } \\
\hline \multirow{2}{*}{$\begin{array}{c}\text { Zone } \\
\text { Comparison }\end{array}$} & \multicolumn{5}{|c|}{ Variable } \\
\hline & CombChromY & AmbChromX1 & AmbCCT1 & AmbCRI1 & IllumWkSfcBrtst \\
\hline K-W Statistic & n.s. & $16.516 ; 0.006$ & $16.516 ; 0.006$ & $17.000 ; 0.004$ & n.s. \\
\hline $1 v 2$ & & & & +++ & \\
\hline 1 v 3 & & --- & --- & +++ & \\
\hline 1 v 4 & & --- & --- & +++ & \\
\hline $1 \vee 5$ & & --- & --- & +++ & \\
\hline 1 v 6 & & --- & --- & +++ & \\
\hline 2 v 3 & & & & +++ & \\
\hline 2 v 4 & & 0.025 & 0.024 & +++ & \\
\hline 2 v 5 & & 0.014 & 0.013 & +++ & \\
\hline 2 v 6 & & & & +++ & \\
\hline 3 v 4 & & --- & --- & +++ & \\
\hline $3 v 5$ & & --- & --- & +++ & \\
\hline 3 v 6 & & --- & --- & +++ & \\
\hline $4 v 5$ & & --- & --- & +++ & \\
\hline $4 \vee 6$ & & -- & -- & +++ & \\
\hline $5 \vee 6$ & & --- & --- & +++ & \\
\hline
\end{tabular}


Table 46. Task 1 Office Buildings Lighting by Zone: Building 005

\begin{tabular}{|c|c|c|c|c|c|}
\hline \multicolumn{6}{|c|}{ ANOVA } \\
\hline \multirow{2}{*}{$\begin{array}{c}\text { Zone } \\
\text { Comparison }\end{array}$} & \multicolumn{5}{|c|}{ Variable } \\
\hline & CombChromX & CombCCT & CombCRI & AmbChromY1 & IllumWkSfcDrkst \\
\hline Main Effect & +++ & $3.850 ; 0.026$ & n.s. & +++ & n.s. \\
\hline $1 \vee 2$ & +++ & & & +++ & \\
\hline $1 \vee 3$ & +++ & & & +++ & \\
\hline $1 v 4$ & +++ & & & +++ & \\
\hline $1 \vee 5$ & +++ & 0.040 & & +++ & \\
\hline $1 \vee 6$ & +++ & & & +++ & \\
\hline 2 v 3 & +++ & & & +++ & \\
\hline $2 \vee 4$ & +++ & & & +++ & \\
\hline $2 v 5$ & +++ & & & +++ & \\
\hline $2 \vee 6$ & +++ & & & +++ & \\
\hline $3 v 4$ & +++ & & & +++ & \\
\hline $3 \vee 5$ & +++ & & & +++ & \\
\hline $3 \vee 6$ & +++ & & & +++ & \\
\hline $4 v 5$ & +++ & 0.035 & & +++ & \\
\hline $4 \vee 6$ & +++ & & & +++ & \\
\hline $5 \vee 6$ & +++ & & & +++ & \\
\hline \multicolumn{6}{|c|}{ Nonparametric Measures } \\
\hline \multirow{2}{*}{$\begin{array}{c}\text { Zone } \\
\text { Comparison }\end{array}$} & \multicolumn{5}{|c|}{ Variable } \\
\hline & CombChromY & AmbChromX1 & AmbCCT1 & AmbCRI1 & IllumWkSfcBrtst \\
\hline K-W Statistic & $17.000 ; 0.004$ & $17.000 ; 0.004$ & n.s. & n.s. & n.s. \\
\hline $1 v 2$ & +++ & +++ & & & \\
\hline $1 \vee 3$ & +++ & +++ & & & \\
\hline $1 \vee 4$ & +++ & +++ & & & \\
\hline $1 \vee 5$ & +++ & +++ & & & \\
\hline $1 \vee 6$ & +++ & +++ & & & \\
\hline 2 v 3 & +++ & +++ & & & \\
\hline 2 v 4 & +++ & +++ & & & \\
\hline 2 v 5 & +++ & +++ & & & \\
\hline $2 \vee 6$ & +++ & +++ & & & \\
\hline 3 v 4 & +++ & +++ & & & \\
\hline $3 v 5$ & +++ & +++ & & & \\
\hline $3 \vee 6$ & +++ & +++ & & & \\
\hline $4 v 5$ & +++ & +++ & & & \\
\hline 4 v 6 & +++ & +++ & & & \\
\hline $5 \vee 6$ & +++ & +++ & & & \\
\hline
\end{tabular}




\section{STATISTICAL ANALYSIS AND RESULTS}

Table 47. Task 1 Office Building Lighting by Zone: Building 007

\begin{tabular}{|c|c|c|c|c|c|}
\hline \multicolumn{6}{|c|}{ ANOVA } \\
\hline \multirow[t]{2}{*}{ Zone Comparison } & \multicolumn{5}{|c|}{ Variable } \\
\hline & CombChromX & CombCCT & CombCRI & AmbChromY1 & IllumWkSfcDrkst \\
\hline Main Effect & n.s. & n.s. & n.s. & +++ & n.s. \\
\hline 1 v 2 & & & & +++ & \\
\hline 1 v 3 & & & & +++ & \\
\hline 1 v 4 & & & & +++ & \\
\hline 1 v 5 & & & & +++ & \\
\hline $1 \vee 6$ & & & & +++ & \\
\hline 2 v 3 & & & & +++ & \\
\hline 2 v 4 & & & & +++ & \\
\hline $2 \vee 5$ & & & & +++ & \\
\hline $2 \vee 6$ & & & & +++ & \\
\hline $3 v 4$ & & & & +++ & \\
\hline $3 v 5$ & & & & +++ & \\
\hline $3 v 6$ & & & & +++ & \\
\hline 4 v 5 & & & & +++ & \\
\hline $4 \vee 6$ & & & & +++ & \\
\hline $5 \vee 6$ & & & & +++ & \\
\hline \multicolumn{6}{|c|}{ Nonparametric Measures } \\
\hline \multirow[t]{2}{*}{ Zone Comparison } & \multicolumn{5}{|c|}{ Variable } \\
\hline & CombChromY & AmbChromX1 & AmbCCT1 & AmbCRI1 & IllumWkSfcBrtst \\
\hline K-W Statistic & n.s. & $17.000 ; 0.004$ & n.s. & n.s. & $11.152 ; 0.048$ \\
\hline 1 v 2 & & +++ & & & \\
\hline $1 v 3$ & & +++ & & & \\
\hline 1 v 4 & & +++ & & & \\
\hline 1 v 5 & & +++ & & & \\
\hline $1 \vee 6$ & & +++ & & & \\
\hline 2 v3 & & +++ & & & \\
\hline $2 v 4$ & & +++ & & & 0.043 \\
\hline $2 v 5$ & & +++ & & & \\
\hline $2 v 6$ & & +++ & & & \\
\hline $3 v 4$ & & +++ & & & 0.033 \\
\hline $3 v 5$ & & +++ & & & \\
\hline $3 \vee 6$ & & +++ & & & \\
\hline $4 v 5$ & & +++ & & & \\
\hline $4 \vee 6$ & & +++ & & & \\
\hline $5 v 6$ & & +++ & & & \\
\hline
\end{tabular}


Table 48. Task 1 Office Buildings Lighting by Zone: Building 008

\begin{tabular}{|c|c|c|c|c|c|}
\hline \multicolumn{6}{|c|}{ ANOVA } \\
\hline \multirow[t]{2}{*}{ Zone Comparison } & \multicolumn{5}{|c|}{ Variable } \\
\hline & CombChromX & CombccT & CombCRI & AmbChromY1 & IllumWkSfcDrkst \\
\hline Main Effect & $3.941 ; 0.024$ & $19.402 ; 0.000$ & $8.846 ; 0.001$ & +++ & n.s. \\
\hline $1 \vee 2$ & & & & +++ & \\
\hline 1 v 3 & & & & +++ & \\
\hline $1 \mathrm{v} 4$ & & 0.000 & & +++ & \\
\hline 1 v 5 & & 0.003 & & +++ & \\
\hline $1 v 6$ & & & & +++ & \\
\hline 2 v 3 & & & & +++ & \\
\hline $2 v 4$ & & 0.001 & & +++ & \\
\hline $2 v 5$ & & & & +++ & \\
\hline 2 v 6 & & & 0.042 & +++ & \\
\hline $3 v 4$ & 0.041 & 0.000 & & +++ & \\
\hline $3 v 5$ & & 0.049 & & +++ & \\
\hline $3 v 6$ & & & 0.042 & +++ & \\
\hline 4 v 5 & & 0.048 & & +++ & \\
\hline $4 \vee 6$ & 0.023 & 0.000 & & +++ & \\
\hline $5 \vee 6$ & & 0.014 & & +++ & \\
\hline \multicolumn{6}{|c|}{ Nonparametric Measures } \\
\hline \multirow[t]{2}{*}{ Zone Comparison } & \multicolumn{5}{|c|}{ Variable } \\
\hline & CombChromY & AmbChromX1 & AmbCCT1 & AmbCRI1 & IllumWkSfcBrtst \\
\hline K-W Statistic & n.s. & n.s. & n.s. & n.s. & $13.757 ; 0.017$ \\
\hline \multicolumn{6}{|l|}{1 v 2} \\
\hline \multicolumn{6}{|l|}{1 v 3} \\
\hline 1 v 4 & & & & & 0.009 \\
\hline 1 v 5 & & & & & 0.046 \\
\hline $1 v 6$ & & & & & 0.001 \\
\hline \multicolumn{6}{|l|}{2 v 3} \\
\hline $2 \vee 4$ & & & & & 0.016 \\
\hline \multicolumn{6}{|l|}{$2 \vee 5$} \\
\hline 2 v 6 & & & & & 0.002 \\
\hline $3 v 4$ & & & & & 0.010 \\
\hline $3 v 5$ & & & & & 0.050 \\
\hline $3 v 6$ & & & & & 0.001 \\
\hline \multicolumn{6}{|l|}{$4 \vee 5$} \\
\hline \multicolumn{6}{|l|}{$4 \vee 6$} \\
\hline $5 \vee 6$ & & & & & \\
\hline
\end{tabular}




\section{STATISTICAL ANALYSIS AND RESULTS}

Table 49. Task 10 Office Building Lighting by Zone: Building 009

\begin{tabular}{|c|c|c|c|c|c|}
\hline \multicolumn{6}{|c|}{ ANOVA } \\
\hline \multirow{2}{*}{$\begin{array}{c}\text { Zone } \\
\text { Comparison }\end{array}$} & \multicolumn{5}{|c|}{ Variable } \\
\hline & CombChromX & CombCCT & CombCRI & AmbChromY1 & IllumWkSfcDrkst \\
\hline Main Effect & n.s. & n.s. & n.s. & +++ & n.s. \\
\hline 1 v 2 & & & & +++ & \\
\hline 1 v 3 & & & & +++ & \\
\hline 1 v 4 & & & & +++ & \\
\hline 1 v 5 & & & & +++ & \\
\hline 1 v 6 & & & & +++ & \\
\hline 2 v 3 & & & & +++ & \\
\hline $2 v 4$ & & & & +++ & \\
\hline $2 v 5$ & & & & +++ & \\
\hline 2 v 6 & & & & +++ & \\
\hline $3 v 4$ & & & & +++ & \\
\hline $3 \vee 5$ & & & & +++ & \\
\hline $3 \vee 6$ & & & & +++ & \\
\hline 4 v 5 & & & & +++ & \\
\hline $4 \vee 6$ & & & & +++ & \\
\hline $5 \vee 6$ & & & & +++ & \\
\hline \multicolumn{6}{|c|}{ Nonparametric Measures } \\
\hline \multirow{2}{*}{$\begin{array}{c}\text { Zone } \\
\text { Comparison }\end{array}$} & \multicolumn{5}{|c|}{ Variable } \\
\hline & CombChromY & AmbChromX1 & AmbCCT1 & AmbCRI1 & IllumWkSfcBrtst \\
\hline K-W Statistic & n.s. & $17.000 ; 0.004$ & $17.000 ; 0.004$ & $17.000 ; 0.004$ & n.s. \\
\hline 1 v 2 & & +++ & +++ & +++ & \\
\hline 1 v 3 & & +++ & +++ & +++ & \\
\hline 1 v 4 & & +++ & +++ & +++ & \\
\hline 1 v 5 & & +++ & +++ & +++ & \\
\hline 1 v 6 & & +++ & +++ & +++ & \\
\hline 2 v 3 & & +++ & +++ & +++ & \\
\hline 2 v 4 & & +++ & +++ & +++ & \\
\hline 2 v 5 & & +++ & +++ & +++ & \\
\hline $2 v 6$ & & +++ & +++ & +++ & \\
\hline $3 v 4$ & & +++ & +++ & +++ & \\
\hline $3 v 5$ & & +++ & +++ & +++ & \\
\hline $3 v 6$ & & +++ & +++ & +++ & \\
\hline $4 \vee 5$ & & +++ & +++ & +++ & \\
\hline $4 \vee 6$ & & +++ & +++ & +++ & \\
\hline $5 v 6$ & & +++ & +++ & +++ & \\
\hline
\end{tabular}


Table 50. Task 13 Schools Lighting by Zone: All Buildings

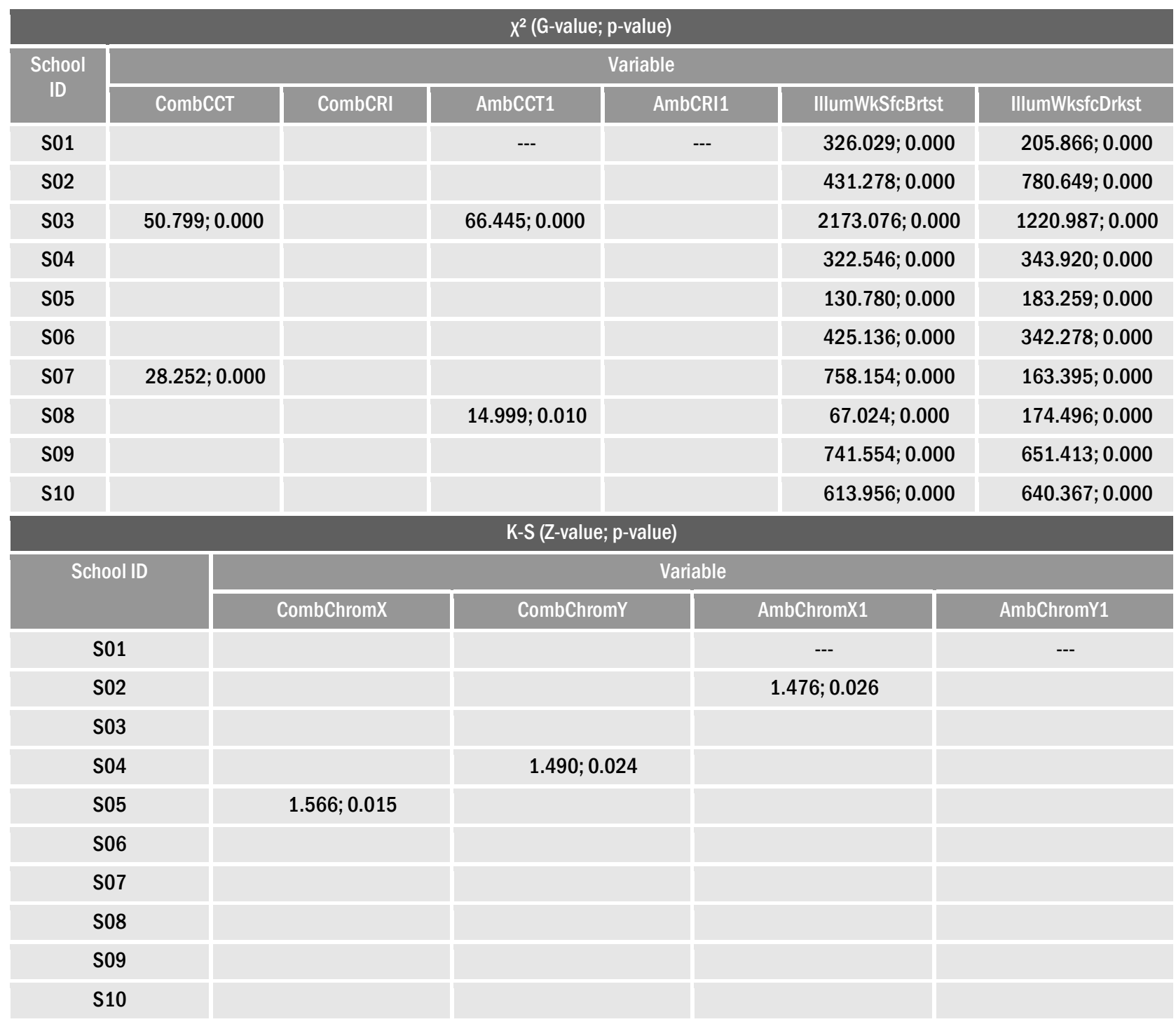


Table 51. Task 0606 High Performance Lighting by Zone: All Buildings

\begin{tabular}{|c|c|c|}
\hline \multicolumn{3}{|c|}{$x^{2}$ (G-value; p-value) } \\
\hline \multirow[t]{2}{*}{ HP Bldg ID } & \multicolumn{2}{|c|}{ Variable } \\
\hline & IllumWkSfcBrtst & IllumWksfcDrkst \\
\hline HPB01 & $282.871 ; 0.000$ & $231.365 ; 0.000$ \\
\hline HPB02 & $87.879 ; 0.000$ & $66.826 ; 0.000$ \\
\hline HPB03 & $386.523 ; 0.000$ & $114.130 ; 0.000$ \\
\hline HPB04 & $713.999 ; 0.000$ & $74.451 ; 0.000$ \\
\hline HPB05 & $1615.865 ; 0.000$ & $330.050 ; 0.000$ \\
\hline HPB06 & $1024.243 ; 0.000$ & $160.329 ; 0.000$ \\
\hline HPB07 & $139.537 ; 0.000$ & $472.492 ; 0.000$ \\
\hline HPB08 & $478.887 ; 0.000$ & $224.591 ; 0.000$ \\
\hline HРB09 & $1756.463 ; 0.000$ & $1078.375 ; 0.000$ \\
\hline HPB10 & $177.501 ; 0.000$ & $228.699 ; 0.000$ \\
\hline
\end{tabular}

\subsection{SOUND}

Sound data sets were previously not presented with the individual measurements for each day, and they were instead aggregated for each zone. This issue was remedied in this task and all tasks sound data were converted to SPSS format for statistical analysis.

Similarly to previous data sets, sound quality was analyzed to compare whether differences in samples collected were apparent both by-day and by-zone. The two variables of interest for all tasks were: cumulative probability levels for dBA at 99\% (L_99_dBA) and at 95\% (L_95_dBA). Univariate analysis of variance (ANOVA) was used to determine the presence or absence of a main effect among the three days for all tasks combined or the six zones for each building within the three tasks. If a main effect was observed, post-hoc analysis was performed to determine which specific days or zones differed through Tukey's Honestly Significant Difference (HSD), Bonferroni's test, or Dunnett's T3; these were for the assumption of equal variance with same sample sizes, equal variance with different sample sizes, or unequal variance, respectively.

A few data points were not collected for a particular zone in the following buildings: in Task 1, Tuesday for Zone 2 in building O04 and Wednesday for Zone 2 of building O10; in Task 13, all Tuesdays for zones 1-6 in building S06; and in Task 0606, all Thursdays for zones 1-6 in building HPB02. Additionally in Task 0606, no data were collected from zones 1, 2, 5, \& 6 of building HPB01. In an effort to retain homogeneity of samples, buildings O04, O10, S06, HPB01, and HPB02 were excluded from comparisons by-day, whereas zone analyses for HPB01 were not conducted. Although no differences were found byday for either L_99_dBA $[\mathrm{F}(2,477)=.08 ; \mathrm{p}=0.92]$ or $\mathrm{L} \_95 \_\mathrm{dBA}[\mathrm{F}(2,477)=0.25 ; \mathrm{p}=0.78]$, significant differences were found among zones for all tasks as shown in the tables (Table 52 - . 
Table 52. Main Effects of Sound Variables for by Building

\begin{tabular}{|c|c|c|c|c|}
\hline \multicolumn{5}{|c|}{ Task 1} \\
\hline \multirow[t]{2}{*}{ Bldg ID } & \multicolumn{2}{|c|}{ L_99_dBA } & \multicolumn{2}{|c|}{ L_95_dBA } \\
\hline & F-value & P-value & F-value & P-value \\
\hline 001 & 12.017 & $<0.001$ & 36.989 & $<0.001$ \\
\hline 002 & 81.686 & $<0.001$ & 42.987 & $<0.001$ \\
\hline 003 & 1.589 & 0.236 & 4.612 & 0.014 \\
\hline 004 & 5.129 & 0.011 & 4.798 & 0.014 \\
\hline 005 & 3.113 & 0.050 & 8.480 & 0.001 \\
\hline 006 & 4.702 & 0.013 & 14.195 & $<0.001$ \\
\hline 007 & 11.396 & $<0.001$ & 23.483 & $<0.001$ \\
\hline 008 & 9.504 & 0.001 & 47.483 & $<0.001$ \\
\hline 009 & 6.290 & 0.004 & 5.986 & 0.005 \\
\hline 010 & 9.619 & 0.001 & 8.445 & 0.002 \\
\hline \multicolumn{5}{|c|}{ Task 13} \\
\hline \multirow[t]{2}{*}{ Bldg } & \multicolumn{2}{|c|}{ L_99_dBA } & \multicolumn{2}{|c|}{ L_95_dBA } \\
\hline & F-value & P-value & F-value & P-value \\
\hline S01 & 2.640 & 0.078 & 1.875 & 0.173 \\
\hline S02 & 7.863 & 0.002 & 6.164 & 0.005 \\
\hline S03 & 7.253 & 0.002 & 7.834 & 0.002 \\
\hline S04 & 4.976 & 0.011 & 5.477 & 0.007 \\
\hline S05 & 0.636 & 0.677 & 0.312 & 0.897 \\
\hline S06 & 12.890 & 0.004 & 29.758 & $<0.001$ \\
\hline S07 & 17.263 & $<0.001$ & 19.600 & $<0.001$ \\
\hline S08 & 0.866 & 0.531 & 0.592 & 0.707 \\
\hline S09 & 9.566 & 0.001 & 14.721 & $<0.001$ \\
\hline S10 & 9.324 & 0.001 & 7.724 & 0.002 \\
\hline \multicolumn{5}{|c|}{ Task 0606} \\
\hline \multirow[t]{2}{*}{ Bldg ID } & \multicolumn{2}{|c|}{ L_99_dBA } & \multicolumn{2}{|c|}{ L_95_dBA } \\
\hline & F-value & P-value & F-value & P-value \\
\hline HPB01 & \multicolumn{4}{|c|}{ Excluded } \\
\hline HPB02 & 9.370 & 0.008 & 9.703 & 0.008 \\
\hline HPB03 & 46.353 & $<0.001$ & 50.595 & $<0.001$ \\
\hline HPB04 & 28.004 & $<0.001$ & 31.030 & $<0.001$ \\
\hline HPB05 & 1.669 & 0.216 & 1.853 & 0.177 \\
\hline HPB06 & 14.348 & $<0.001$ & 14.390 & $<0.001$ \\
\hline HPB07 & 3.167 & 0.047 & 3.055 & 0.052 \\
\hline HРB08 & 22.890 & $<0.001$ & 24.930 & $<0.001$ \\
\hline HPB09 & 8.233 & 0.001 & 9.398 & 0.001 \\
\hline HPB10 & 68.208 & $<0.001$ & 57.686 & $<0.001$ \\
\hline
\end{tabular}


Table 53. L_99_dBA for Task 1 by Zone

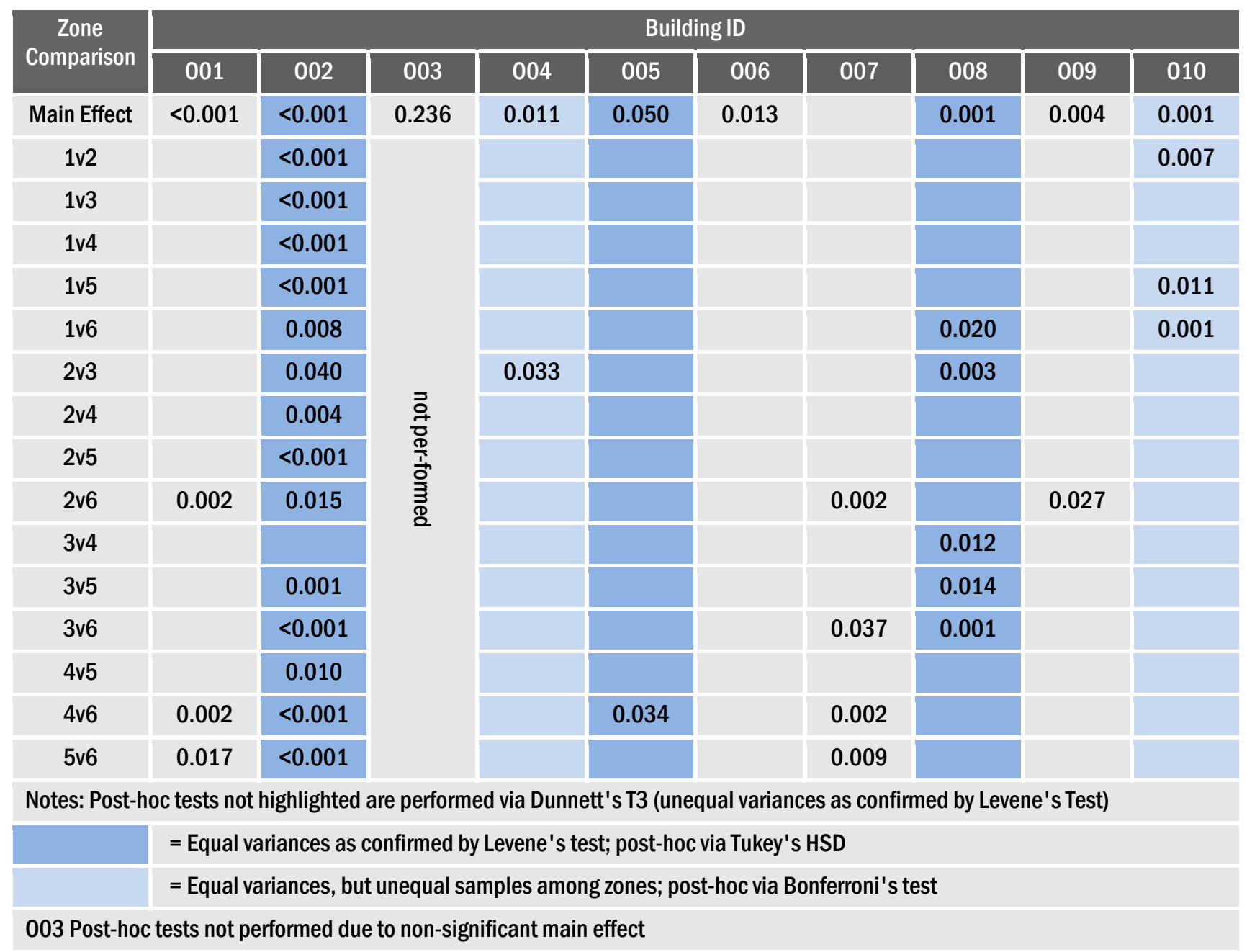


Table 54. . L_95_dBA for Task 1 by Zone

\begin{tabular}{|c|c|c|c|c|c|c|c|c|c|c|}
\hline \multirow{2}{*}{$\begin{array}{c}\text { Zone } \\
\text { Comparison }\end{array}$} & \multicolumn{10}{|c|}{ Building ID } \\
\hline & 001 & 002 & 003 & 004 & 005 & 006 & 007 & 008 & 009 & 010 \\
\hline Main Effect & $<0.001$ & $<0.001$ & 0.014 & 0.014 & & $<0.001$ & $<0.001$ & $<0.001$ & 0.005 & 0.002 \\
\hline $1 v 2$ & & $<0.001$ & & & & 0.005 & & & & 0.010 \\
\hline $1 v 3$ & & 0.001 & & & & & & & & \\
\hline $1 v 4$ & & $<0.001$ & & & & 0.006 & & 0.039 & & \\
\hline $1 v 5$ & & $<0.001$ & & & & & & 0.039 & & \\
\hline $1 v 6$ & & & & & & & & 0.017 & & 0.005 \\
\hline $2 v 3$ & & & & & & 0.049 & & & & \\
\hline $2 v 4$ & & & & & & 0.001 & & & & 0.048 \\
\hline $2 v 5$ & & 0.018 & & & & & & & & \\
\hline $2 v 6$ & 0.001 & $<0.001$ & & & 0.021 & & 0.014 & & 0.036 & \\
\hline $3 v 4$ & & & 0.008 & & & & & 0.003 & & \\
\hline $3 v 5$ & & 0.001 & 0.040 & & & & & 0.042 & & \\
\hline $3 v 6$ & 0.010 & 0.001 & & & 0.027 & & 0.033 & 0.001 & 0.031 & \\
\hline $4 v 5$ & & 0.009 & & & & & 0.041 & & & \\
\hline $4 v 6$ & $<0.001$ & $<0.001$ & & & & & 0.004 & 0.001 & & 0.026 \\
\hline $5 v 6$ & 0.022 & $<0.001$ & & & & & 0.001 & & & \\
\hline \multicolumn{11}{|c|}{ Notes: Post-hoc tests not highlighted are performed via Dunnett's T3 (unequal variances as confirmed by Levene's Test) } \\
\hline & \multicolumn{10}{|c|}{ = Equal variances as confirmed by Levene's test; post-hoc via Tukey's HSD } \\
\hline & \multicolumn{10}{|c|}{ = Equal variances, but unequal samples among zones; post-hoc via Bonferroni's test } \\
\hline
\end{tabular}


Table 55. L_99_dBA for Task 13 by Zone

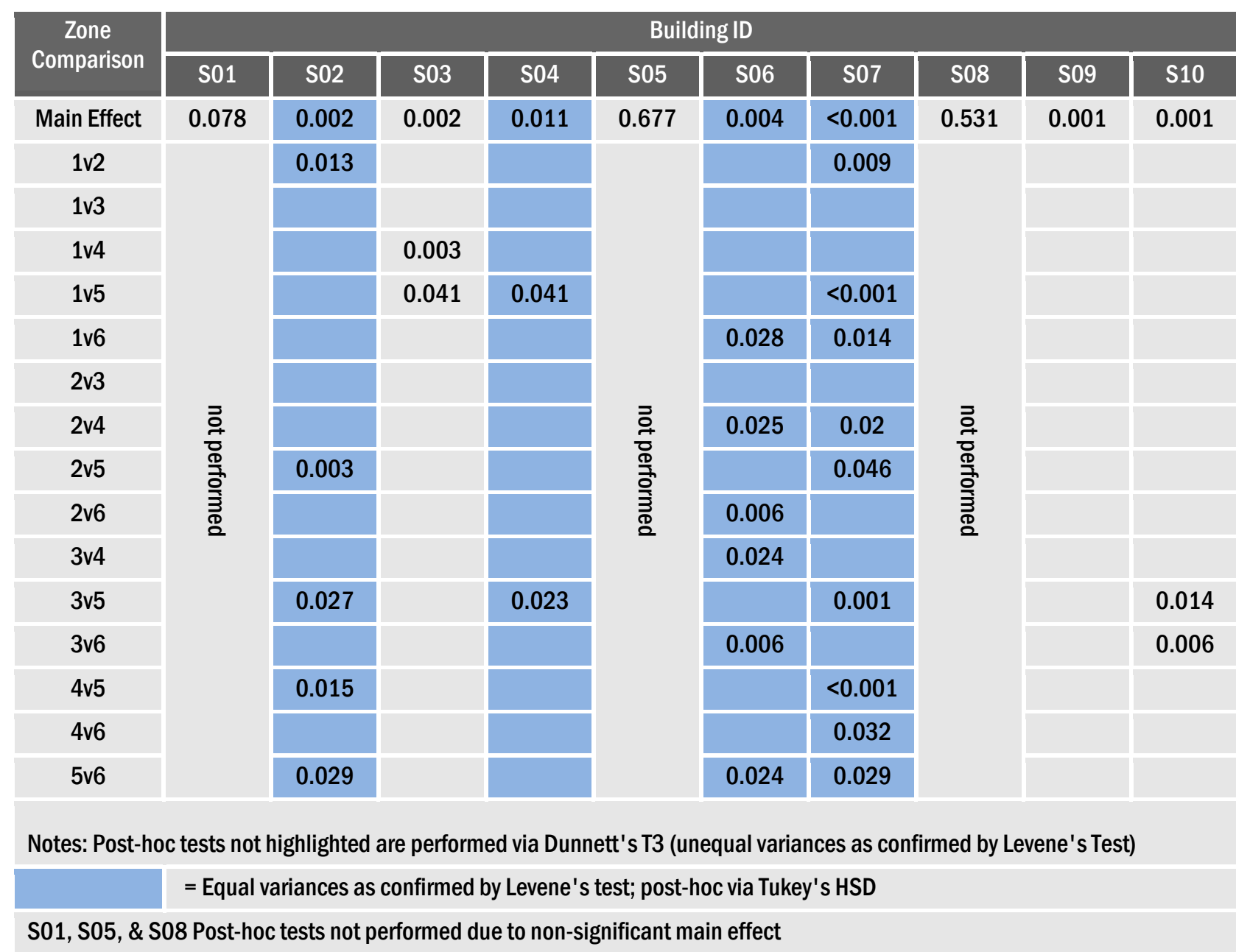


Table 56. L_95_dBA for Task 13 by Zone

\begin{tabular}{|c|c|c|c|c|c|c|c|c|c|c|}
\hline \multirow{2}{*}{$\begin{array}{c}\text { Zone } \\
\text { Comparison }\end{array}$} & \multicolumn{10}{|c|}{ Building ID } \\
\hline & S01 & S02 & S03 & S04 & S05 & S06 & S07 & S08 & S09 & S10 \\
\hline Main Effect & 0.173 & 0.005 & 0.002 & 0.007 & 0.897 & $<0.001$ & $<0.001$ & 0.707 & $<0.001$ & 0.002 \\
\hline 1v2 & \multirow{15}{*}{ 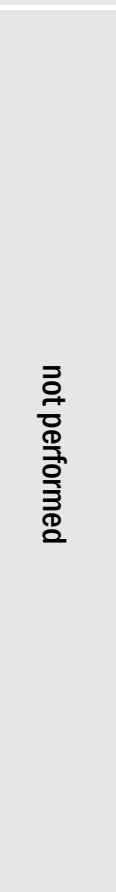 } & 0.025 & & & \multirow{15}{*}{ 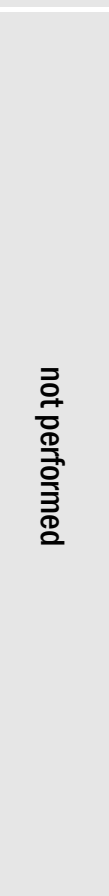 } & & 0.003 & \multirow{15}{*}{ 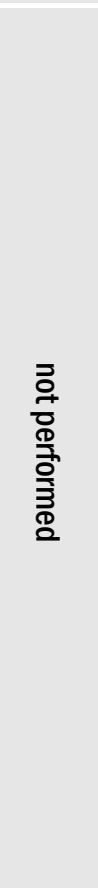 } & & \\
\hline $1 v 3$ & & & & & & & & & & \\
\hline $1 v 4$ & & & 0.007 & & & 0.018 & & & & \\
\hline $1 v 5$ & & & 0.039 & 0.023 & & & $<0.001$ & & & \\
\hline $1 v 6$ & & & 0.027 & & & 0.003 & 0.008 & & & \\
\hline \multicolumn{8}{|l|}{ 2v3 } & & & \\
\hline $2 \mathrm{v} 4$ & & & 0.007 & & & 0.002 & 0.008 & & & \\
\hline $2 \mathrm{v} 5$ & & 0.005 & 0.039 & 0.033 & & & & & & \\
\hline $2 v 6$ & & & 0.027 & & & 0.001 & & & & \\
\hline $3 v 4$ & & & & & & 0.005 & & & & \\
\hline $3 v 5$ & & 0.050 & & 0.018 & & & $<0.001$ & & & 0.009 \\
\hline $3 v 6$ & & & & & & 0.001 & & & 0.037 & 0.004 \\
\hline $4 v 5$ & & 0.043 & & & & 0.013 & $<0.001$ & & & \\
\hline $4 v 6$ & & & & & & & 0.019 & & & \\
\hline $5 v 6$ & & & & & & 0.002 & 0.027 & & & \\
\hline Notes: Post-h & Lesto IIV & ghlighted & re perforr & d via Dun & tt's T3 (u & qual varia & ces as con & ned by & ene's Test & \\
\hline & $=$ Equal & iances as & onfirmed & Levene's & st; post- & c via Tuke & s HSD & & & \\
\hline
\end{tabular}


Table 57. L_99_dBA for Task 0606 by Zone

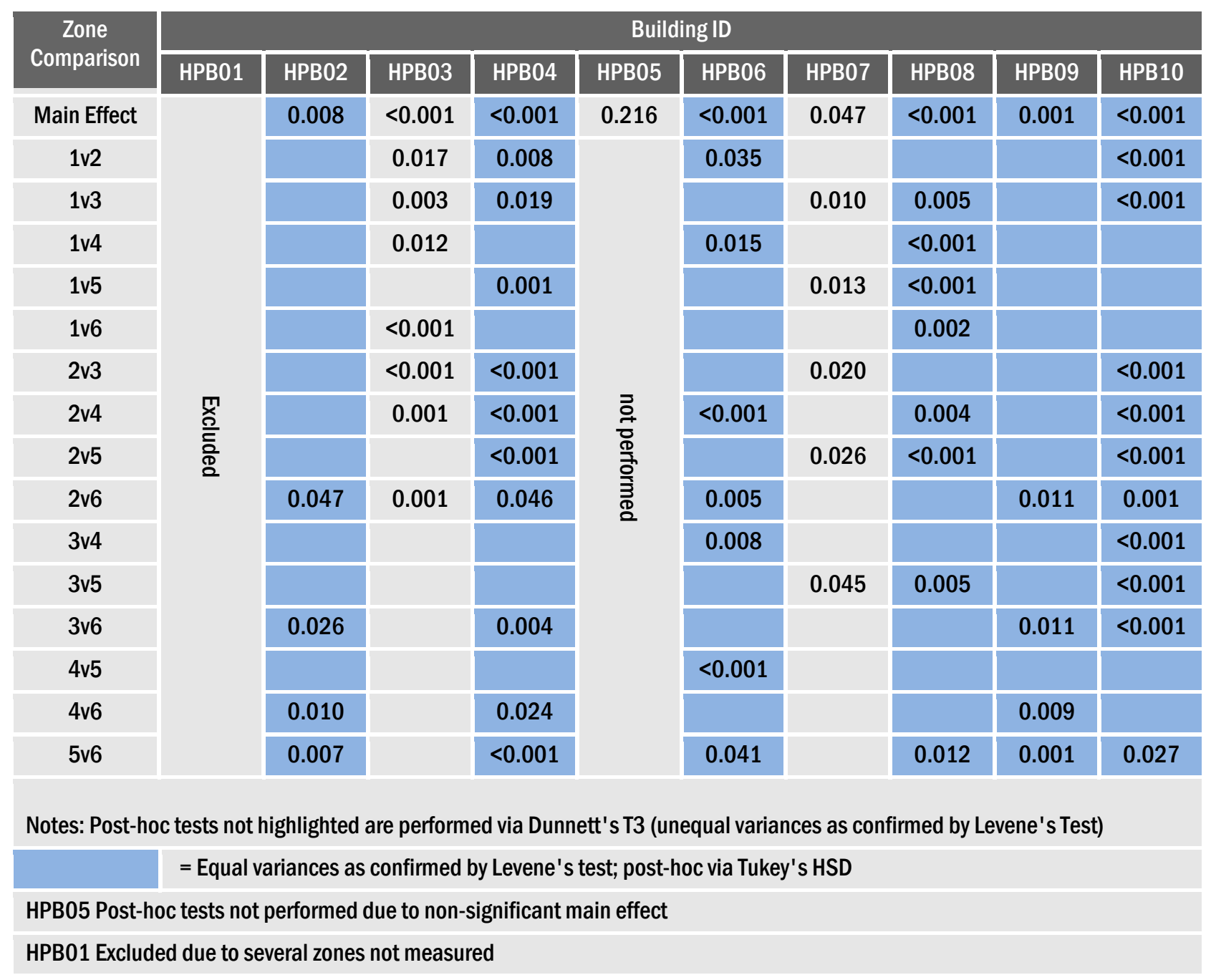


Table 58. L_95_dBA for Task 0606 by Zone

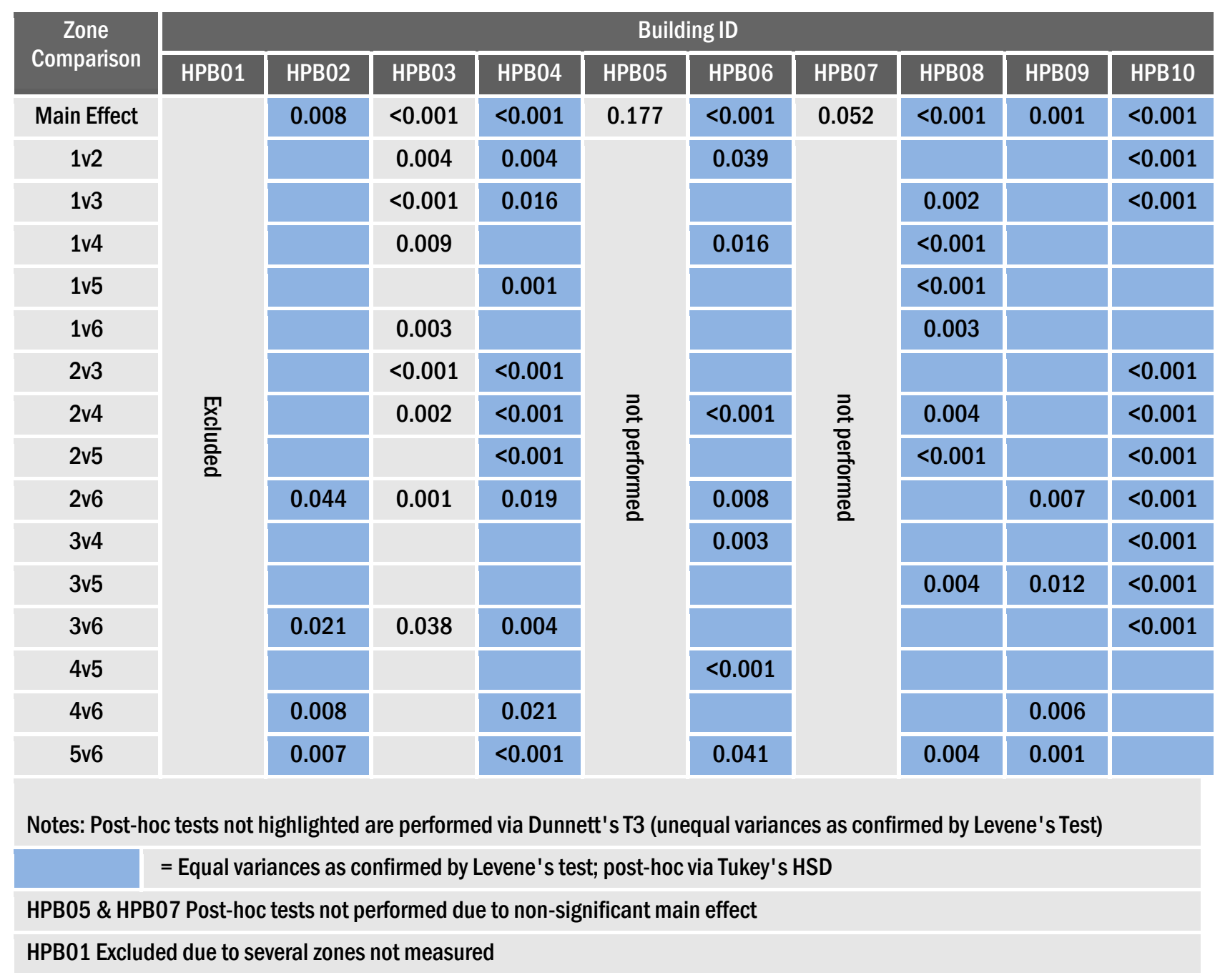




\section{INTERPRETATION OF THE DATA}

This task was focused on interpretation of the statistical analysis to provide conclusions as to the robustness of the monitoring package in providing reliable building measurement data with a finite number of monitoring stations and a limited time frame for monitoring. It is expected that this interpretation will increase the utility of the data and provide previously available information.

\subsection{IEQ}

Table 1 demonstrated that Thursday measures were significantly different from Tuesday, and most Wednesday measures for all variables of Tasks $1 \& 13$. Data for all three days appeared to vary for Operative Temperature, whereas only Tuesday and Thursday differed for Draft Rate $1 \mathrm{~m}$. Although no Draft Rate measures within Task 0606 were found to significantly differ by-day, Temperature measurements for Tuesday and Thursday showed variation. Moreover, Wednesday measurements of Relative Humidity within Task 0606 varied significantly from either Tuesday or Thursday.

As shown in Tables 2-31, the majority of differences by-zone were highly significant $(p<0.001)$, with the exception of Relative Humidity in some buildings. Although all zone comparisons are listed in the tables, several zones were excluded due to lack of collection of measurements for one of the zones. For example, if no data were acquired for Zone 3 of a particular building, then all post-hoc analyses against Zone 3 were excluded. Additionally, Bldg 02 zone 1 of Task 0606 only contained two points of data, and thus was excluded from all variable comparisons.

\subsection{AIRBORNE AND SURFACE-ASSOCIATED MOLD}

When all task data were combined (30 buildings) neither the airborne culturable fungi nor the airborne non-culturable spore data differed in the concentrations found at any indoor location in terms of day of collection. However, the concentrations of surface-associated fungi varied among the day of collection. Specifically, there was a lower concentration of mold on Tuesday than on Wednesday, for all tasks combined.

As expected, variation was found in the concentrations of both airborne culturable fungi and airborne non-culturable fungal spores between indoor zones (1-6) and the outdoor zone (zone 0). No variation was found among the indoor zones of office buildings for Task 1 in the concentrations of airborne culturable fungi. However, airborne non-culturable spores did vary among zones in one building in Task 1 and variation was noted between zones in surface-associated fungi.

\subsection{LIGHTING}

Due to the lack of multiple measurements for Tasks 13 and 0606, by-day comparisons were only performed for Task 1. No statistical differences were observed with respect to the day of collection. There was a wide range of variability by-zone among seven of the office buildings. Although few differences were found for IllumWkSfcBrtst and IllumWkSfcDrkst in Task 1, there was a great amount of variation for these variables in Tasks 13 and $0606(\mathrm{p}<0.001)$. Other variables that differed by-zone in Task 13 include CombCCT and AmbCCT1 for S03, S07, and S08. Additionally, AmbChromX1, CombChromY, and CombChromX varied by-zone for school buildings S02, S04, and S05, respectively. 


\subsection{SOUND}

Tables 52a-52c provide an overview of the variability among each building within each task. Although all tasks demonstrated significant differences by-zone, some of the buildings within each task did not appear to differ in sound quality. Hence, post-hoc tests were not appropriate and individual zones were not compared for these buildings. As shown in Tables 55 and 56, buildings S01, S05, and S08 do not provide further information beyond the p-value of the main effect.

It is interesting to note that some buildings were widely varied with most zone comparisons and other buildings varied between only a few zones. For instance, Table 53 shows that the difference between zones for O02 were highly significant except in the comparison of zones 3 and 4 . Whereas, for O04, only zones 2 and 3 differed from each other. Another pattern observed was a major difference of a single zone as compared to all other zones. As can be seen in Table 58, of the zones in HPB02, only zone 6 appeared to differ from the other zones with the exception of zone 1 . 


\section{JOURNAL ARTICLE}

This task was focused on the submission of an article to a peer reviewed journal describing the results of the statistical analysis and interpretation, and recommendations for a reliable monitoring strategy for assessing indoor environmental quality in the built environment. This journal article will provide dissemination of previously unavailable information on energy usage, building characteristics and indoor environmental quality measurement data in buildings across the United States.

A journal article detailing the results of the mold data is in the final stages before submission to a peerreviewed journal focusing on indoor air quality and monitoring issues. 


\section{REFERENCES}

Stetzenbach, LC et al. 2008. Measurement and Verification of Building Performance Characteristics. Final Report NCEMBT-080201. Alexandria, Virginia: National Center for Energy Management and Building Technologies.

Stetzenbach, LC et al. 2008b. Energy Performance and Environmental Characteristics of Educational Facilities. Final Report NCEMBT-081208. Alexandria, Virginia: National Center for Energy Management and Building Technologies.

Stetzenbach, LC et al. 2009 (to be published). Building Performance Characterization, Energy Usage, and Indoor Environmental Quality in High Performance Buildings. Final Report NCEMBT-091201. Alexandria, Virginia: National Center for Energy Management and Building Technologies. 
National Center for Energy Management and BuILding TeChnologies

601 NORTH FAIRFAX StREET, SUITE 250

\section{ALEXANDRIA, VA 22314}

WWW.NCEMBT.ORG 